\title{
Cool and hot emission in a recurring active region jet ${ }^{\star}$
}

\author{
Sargam M. Mulay, Giulio Del Zanna, and Helen Mason
}

\begin{abstract}
DAMTP, Centre for Mathematical Sciences, University of Cambridge, Wilberforce Road, Cambridge, CB3 0WA, UK
e-mail: smm96@cam.ac.uk
\end{abstract}

Received 12 January 2017 / Accepted 28 June 2017

\begin{abstract}
Aims. We present a thorough investigation of the cool and hot temperature components in four recurring active region jets observed on July 10, 2015 using the Atmospheric Imaging Assembly (AIA), X-ray Telescope (XRT), and Interface Region Imaging Spectrograph (IRIS) instruments.

Methods. A differential emission measure (DEM) analysis was performed on areas in the jet spire and footpoint regions by combining the IRIS spectra and the AIA observations. This procedure better constrains the low temperature DEM values by adding IRIS spectral lines. Plasma parameters, such as Doppler velocities, electron densities, nonthermal velocities and a filling factor were also derived from the IRIS spectra.

Results. In the DEM analysis, significant cool emission was found in the spire and the footpoint regions. The hot emission was peaked at $\log T[\mathrm{~K}]=5.6-5.9$ and 6.5 respectively. The DEM curves show the presence of hot plasma $(T=3 \mathrm{MK})$ in the footpoint region. We confirmed this result by estimating the Fe XVIII emission from the AIA $94 \AA$ channel which was formed at an effective temperature of $\log T[\mathrm{~K}]=6.5$. The average XRT temperatures were also found to be in agreement with $\log T[\mathrm{~K}]=6.5$. The emission measure (EM) was found to be three orders of magnitude higher in the AIA-IRIS DEM compared with that obtained using only AIA. The O IV (1399/1401 $\AA$ ) electron densities were found to be $2.0 \times 10^{10} \mathrm{~cm}^{-3}$ in the spire and $7.6 \times 10^{10} \mathrm{~cm}^{-3}$ in the footpoint. Different threads along the spire show different plane-of-sky velocities both in the lower corona and transition region. Doppler velocities of $32 \mathrm{~km} \mathrm{~s}^{-1}$ (blueshifted) and $13 \mathrm{~km} \mathrm{~s}^{-1}$ (redshifted) were obtained in the spire and footpoint, respectively from the Si IV $1402.77 \AA$ spectral line. Nonthermal velocities of 69 and $53 \mathrm{~km} \mathrm{~s}^{-1}$ were recorded in the spire and footpoint region, respectively. We obtained a filling factor of 0.1 in the spire at $\log T[\mathrm{~K}]=5$.

Conclusions. The recurrent jet observations confirmed the presence of significant cool emission co-spatial with the coronal emission.
\end{abstract}

Key words. Sun: corona - Sun: atmosphere - Sun: transition region - Sun: UV radiation

\section{Introduction}

The discovery of solar jets at X-ray wavelengths with $\mathrm{YOHKOH}$ (Tsuneta et al. 1991) gave a new perspective to the study of dynamic activity in the corona. These transient events have been observed to originate in quiet Sun regions (Wang et al. 1998; Martínez Pillet et al. 2011; Rubio da Costa et al. 2015), in coronal holes (known as CH jets; Culhane et al. 2007b; Cirtain et al. 2007; Kamio et al. 2007; Nisticò et al. 2011; Young \& Muglach 2014a,b; Chandrashekhar et al. 2014) and also from the periphery of active regions (known as AR jets; Raouafi et al. 2016). The AR jets are mostly associated with sunspot regions and their signatures have been observed in $\mathrm{H} \alpha$ (Canfield et al. 1996; Jiang et al. 2007; Chen et al. 2008; Wang et al. 2014; Hong et al. 2017), Ca II k (Shibata et al. 2007); at UV/EUV wavelengths (Innes et al. 2011; Schmieder et al. 2013; Zhang \& Ji 2014a; Chandra et al. 2015; Mulay et al. 2016; Sterling et al. 2016; Innes et al. 2016; Joshi et al. 2017) and X-ray wavelengths (Shibata et al. 1992; Shimojo et al. 1996; Kim et al. 2007; Chifor et al. 2008a,b; Mulay et al. 2017).

Substantial progress has been made in last few decades to understand the initiation mechanism and plasma parameters of jets using high-resolution observations and 2D/3D numerical simulations. The observations and numerical simulation confirm that

\footnotetext{
$\star$ The movie attached to Fig. 3 is available at http://www . aanda.org
}

magnetic reconnection is the principle mechanism responsible for the ejection of jets. The emergence of new magnetic flux in the photosphere and its interaction with pre-existing magnetic field changes the topology of the magnetic field in the corona and as a result collimated plasma ejects from the reconnection site and often appears as an inverted Y-shape jet (Shibata et al. 1992; Shibata et al. 1994; Yokoyama \& Shibata 1995). Also, some jets have been observed to occur in magnetic flux cancellation events (Hong et al. 2011; Adams et al. 2014; Young \& Muglach 2014a,b; Panesar et al. 2016b; Sterling et al. 2017).

More recent space-based instruments such as the Atmospheric Imaging Assembly (AIA; Lemen et al. 2012) onboard the Solar Dynamic Observatory, the X-ray Telescope (XRT; Golub et al. 2007), the EUV Imaging Spectrometer (EIS; Culhane et al. 2007a) onboard the Hinode satellite (Kosugi et al. 2007), and the Interface Region Imaging Spectrograph (IRIS; De Pontieu et al. 2014) are advancing our understanding of AR jets by enabling us to probe different temperatures of the solar atmosphere.

With high resolution and high cadence observations from the AIA, it is possible to follow the evolution of AR jets in different UV and EUV passbands which are sensitive to multiple temperatures. The jets which have been observed to recur at the same place at the edge of ARs which are known as recurrent AR jets (Schmieder et al. 2013; Chandra et al. 2015; Mulay et al. 2017) and in some cases, they show similar morphology and direction of plasma outflow (then known as homologous AR jets; 
Zhang \& Ji 2014b; Yu-kun et al. 2016; Liu et al. 2016). In this paper, we shall focus on AR jet observations.

Since these AR jets have been observed over a wide range of wavelengths from $\mathrm{H} \alpha$ to X-rays, it is important to study the temperature structure of these transient events, their relationship and behaviour in different layers of solar atmosphere. A number of authors have studied the temperature structure using a differential emission measure (DEM) analysis. These analyses are based on the assumption that multithermal plasma is present along the line-of-sight. Using six AIA EUV channels (94, 131, 171, 193, 211 and $335 \AA$ ) which are sensitive to coronal temperatures, a number of authors (Chen et al. 2013; Kayshap et al. 2013; Mulay et al. 2016; Liu et al. 2016) have found a peak temperature of 1-3 MK using different DEM analysis codes (Hannah \& Kontar 2012; Aschwanden et al. 2013)

In a recent study of AR jets, Mulay et al. (2017) presented a direct comparison of spectroscopic (Hinode/EIS) and imaging (SDO/AIA, Hinode/XRT) observations of AR jets. The authors obtained the temperature of the jet's spire and the footpoint region separately from the EIS spectra and also by combining AIA and XRT data. The purpose of using the XRT data together with the AIA data in the DEM analysis was to constrain the high temperature part in the DEM curves. They showed that the EIS DEM values were in good agreement with those obtained from the AIA-XRT data in the temperature range from $\log T[\mathrm{~K}]=5.9-6.3$. They obtained the same peak temperature $T=2.0 \mathrm{MK}(\log T[\mathrm{~K}]=6.3)$ in both regions (spire and footpoint).

The EIS observations had Mg VII $(\log T[\mathrm{~K}]=5.7)$ and Fe XV $(\log T[\mathrm{~K}]=6.4)$ lines to constrain the low and high temperatures respectively, in the DEM analysis. However, the DEM curves were not well constrained below $\log T[\mathrm{~K}]=5.7$ and above $\log T[\mathrm{~K}]=6.4$ which is evident from large error bars (see Mulay et al. (2017), Fig. 9). Also, the AIA-XRT DEM for the spire region showed large error bars below $\log T[\mathrm{~K}]=5.9$ and above $\log T[\mathrm{~K}]=6.4$. The authors reported plasma temperature $T>2 \mathrm{MK}$ in the footpoint region from the AIA-XRT DEM curve and these results were confirmed by estimating the Fe XVIII emission from the AIA $94 \AA$ channel.

A direct comparison of the hot $(\log T[\mathrm{~K}]>6.0)$ and cool $(\log T[\mathrm{~K}]<5.7)$ temperature components in AR jets has not yet been carried out using both spectra and imaging observations, in spite of its importance. This is due to the difficulty of getting simultaneous and co-spatial observations. In fact, different simulations and theoretical ideas on how jets form predict a different behaviour of the two components. For example, if the jet is formed by reconnection in the upper chromosphere, one would expect a hot jet in X-ray wavelengths along with a cool jet in the chromosphere (Shibata et al. 2007).

AR jets are dynamic events which are hard to observe simultaneously with both imaging and spectroscopic instruments. With the launch of IRIS, it is now possible to probe the upper chromosphere and transition region with a spectrograph and slit-jaw imaging instrument which allows us to study the cool thermal component associated with AR jets. The SDO/AIA instrument provides simultaneous monitoring of the higher temperature emission.

The first high resolution observation of a recurrent AR jet from IRIS was reported by Cheung et al. (2015). The spectroscopic observations showed oppositely directed components moving with Doppler velocities of $\pm 100 \mathrm{~km} \mathrm{~s}^{-1}$ indicating a helical motion. The authors obtained electron number densities $\log N_{\mathrm{e}}=10.8-11.2 \mathrm{~cm}^{-3}$ in jets from the O IV $(1399 / 1401 \AA)$ line ratio diagnostic.

Liu et al. (2016) studied a recurrent AR jet originating from the NOAA AR 12381 on July 9, 2015. The authors calculated physical parameters of 11 jets such as the average temperature (1.2-1.5 MK), average electron number density $N_{\mathrm{e}}=0.7-1.1 \times 10^{9} \mathrm{~cm}^{-3}$, and axial speed $\left(383 \pm 9 \mathrm{~km} \mathrm{~s}^{-1}\right)$ from the AIA observations.

Following our previous work on AR jets (Mulay et al. 2016; Mulay et al. 2017), we had been looking for AR jet data simultaneously observed by imaging and spectroscopic instruments, to carry out a more detail analysis of their temperature structure. We found a suitable on-disk (close to the Sun centre) recurrent AR jet, observed on July 10, 2015 simultaneously by IRIS, AIA and XRT. By a coincidence, it was the same active region studied by Liu et al. (2016) a day before on July 9, 2015.

In this paper, we present the first thorough investigation of the cool and hot temperature component observed in a recurrent AR jet using both spectroscopic observations from IRIS spectrograph and imaging observations from the Slit-jaw/IRIS, AIA and XRT instruments. We have performed a DEM analysis by combining spectra from the IRIS with EUV images from AIA and we have compared our results with temperatures obtained from XRT. We believe this to be the first time that such a comprehensive analysis has been carried out for an AR jet.

In Sect. 2, we present the observations and the initial data processing. Section 3 gives details about image co-alignment, density diagnostics and the DEM analysis. In Sect. 4, we discuss and summarize our results and compare them with previous results available in the literature.

\section{Observations}

In this section, we discuss the observations and data processing of a recurrent active region jet observed on July 10, 2015.

\subsection{Atmospheric Imaging Assembly (AIA)}

The AIA instrument observed a recurrent jet on July 10, 2015 (see movie) with high spatial (about $\sim 1.2^{\prime \prime}$ resolution, $0.6^{\prime \prime}$ per pixel) and temporal (12 s) resolution. From 07:29 to 08:35 UT, all EUV/UV channels observed four recurrent jets originating from the eastern edge of AR NOAA 12381 (N14 W25).

The AIA level 1.0 full disk data were downloaded using the Solarsoft VSO package. The AIA EUV/UV channels are dominated by many strong spectral lines (listed below) formed at different temperatures indicative of the multi-thermal nature of the filters (O'Dwyer et al. 2010; Del Zanna et al. 2011; Del Zanna 2013, 2015): $94 \AA$ (Fe X, Fe XIV, Fe XVIII), $131 \AA$ (Fe VIII, Fe XXI), $171 \AA$ (Fe IX), $193 \AA$ (Fe XII, Ca XVII, Fe XXIV), $211 \AA$ (Fe XIV), $304 \AA$ (He II), $335 \AA$ (Fe XVI), $1600 \AA$ (C IV+continuum) and $1700 \AA$ (continuum). The data were processed to level 1.5 using the standard AIA software package (aia_prep.pro) available in the SolarSoft (SSW; Freeland \& Handy 1998) libraries. The intensities in the UV and EUV channels were normalized by the exposure time.

\section{2. $X$-ray Telescope (XRT)}

The XRT imaging instrument observed the recurrent jet in X-ray wavelengths using the Al-poly and Be-thin filters. We obtained high resolution $\left(1.02^{\prime \prime}\right)$ images from these filters with a cadence of $90 \mathrm{~s}$. The X-ray data is available from 07:30 to 07:55 UT and 
Table 1. IRIS observation details.

\begin{tabular}{lcc}
\hline \hline IRIS & Spectrograph & Slit-Jaw-Imager (SJI) \\
\hline Observation ID & 3660106173 & $07: 29: 18$ \\
Start time (UT) & $07: 29: 17$ & $08: 35: 01$ \\
End time (UT) & $08: 35: 15$ & 240 (no. of images) \\
No. of rasters & 4 & \\
No. of slit positions & 192 & $0.33^{\prime \prime}$ \\
Step cadence (s) & 5.2 & $167^{\prime \prime} \times 175^{\prime \prime}$ \\
spatial resolution & $0.33^{\prime \prime}($ slit width) & 4 \\
Field-of-view & $63^{\prime \prime} \times 175^{\prime \prime}$ & 4 \\
Exposure time (s) & 16 min (for each raster) & $17 \mathrm{~s}$ (for C II, Si IV, Mg II) \\
Cadence & & $82 \mathrm{~s}(2832$ window) \\
\end{tabular}

some images are available after 08:34 UT for both filters with a 5 min gap from 07:43 to 07:49 UT in Be-thin filter observation. The XRT data were downloaded and processed using the standard XRT software package (xrt_prep.pro; Kobelski et al. 2014) available in the SSW libraries. In this analysis, we used the updated XRT filter calibration (Narukage et al. 2011). The intensities in each filter images were normalized by the exposure time.

\subsection{Interface Region Imaging Spectrograph (IRIS)}

The IRIS provides simultaneous images and spectra of the solar atmosphere in the far-UV (FUV:1331.56-1358.40 and 1390.00$1406.79 \AA$ ) and near-UV (NUV:2782.56-2833.89 A) wavelength bands with an unprecedented high temporal (2 s), spatial $\left(0.33^{\prime \prime}\right)$ and spectral (26 m $\AA$ (FUV), $53 \mathrm{~m} \AA$ (NUV)) resolution. IRIS observes plasma which forms over a wide range of temperatures (between $5000 \mathrm{~K}$ and $10 \mathrm{MK}$ ) and provides information about the continuum and emission lines in the photosphere, chromosphere, transition region, and corona. The IRIS Slit Jaw Imager (SJI) provides images in four different passbands (C II 1330, Si IV 1400, Mg II k 2796, and Mg II wing $2830 \AA$ A) and the IRIS Spectrograph observes spectral lines formed in the chromosphere (Mg II h $2803 \AA$ and Mg II k $2796 \AA$ ) and transition region (C II 1334/1335 $\AA$ and Si IV 1394/1403 $\AA$ ) and also in the passbands from 1332-1358 ̊, 1389-1407 $\mathrm{A}$, and 2783-2834 $\AA$.

On July 10, 2015, the spectrograph observed AR NOAA 12381 (N14 W25) with 192 steps (dense raster) from 07:29 to 08:35 UT. For each raster observation, the $0.33^{\prime \prime}$ slit was scanning in the direction from east to west (the same as the solar rotation) in $\sim 16 \mathrm{~min}$, covering a field-of-view (FOV) of $63^{\prime \prime} \times 175^{\prime \prime}$ on the solar disk with an exposure time of $4 \mathrm{~s}$. This study included 9 spectral windows (FUV:C II 1336, 1343, Fe XII 1349, O I 1356, Si IV 1403 and NUV:2814, 2826, 2832, Mg II k 2796). Also, the SJI captured 240 frames during this period in four passbands with high spatial $\left(0.33^{\prime \prime}\right)$ and temporal (17 s) resolution. Table 1 provides details of the IRIS observations.

Figure 1 shows a composite image of AR NOAA 12381 in the AIA 171(red), 193(green) and 211(blue) A passbands observed on July 10, 2015 at 08:26 UT. The white box shows the SJI FOV and the yellow box shows the spectrograph FOV. The jet's spire and the footpoint regions are shown by white arrows.

We obtained IRIS level 2 data for both instruments from the data archive available ${ }^{1}$. The level 2 data is prepared from level 1.5 after correcting the dark current, flat-field, geometric and wavelength calibration and FUV background subtraction using the standard routine iris_prep.pro (version 1.49) available

http://iris.lmsal.com/search/

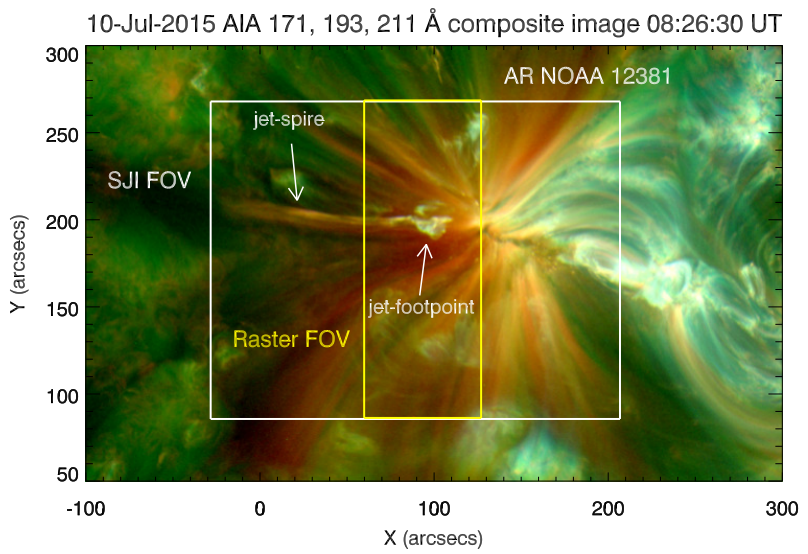

Fig. 1. Active region field-of-view observed by AIA and IRIS. AIA 171(red), 193(green), 211(blue) $\AA$ composite image of active region NOAA 12381 (N14 W25) observed on July 10, 2015. The white box shows the IRIS-SJI field-of-view and the yellow box shows the IRIS spectrograph field-of-view. The white arrows show the spire and the footpoint regions of the jet.

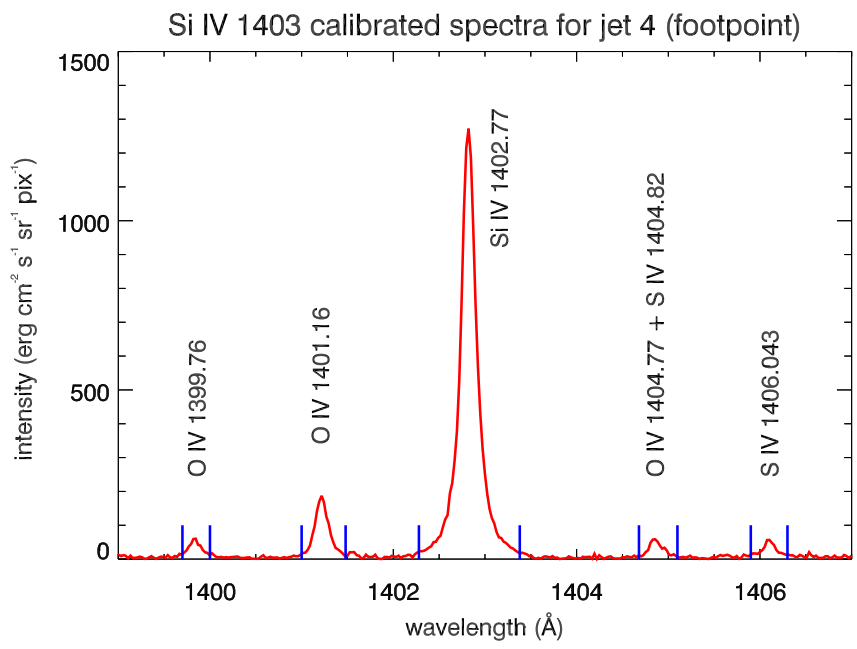

Fig. 2. IRIS spectra for the footpoint region of jet. The calibrated spectra (in erg cm ${ }^{-2} \mathrm{~s}^{-1} \mathrm{sr}^{-1}$ pix $^{-1}$ units) obtained for the Si IV $1403 \AA$ window for the footpoint region of the jet observed in the fourth raster scan (shown as a small yellow box in Fig. 8). The blue vertical lines show the wavelength ranges over which we obtained the total counts for each line.

in the SSW. The level 2 data is also corrected for the orbital variation (both the thermal component and the spacecraft velocity component) using the iris_orbitvar_corr_12s.pro routine. The dark regions seen in the SJI FUV images (because of accumulation of dust on the CCD) are removed using the iris_dustbuster.pro routine and the cosmic rays were removed using the despik. pro routine.

Figure 2 shows the calibrated spectra for the Si IV $1403 \AA$ window obtained for the footpoint region of the jet observed in the fourth raster scan. The spectra were created using the iris_sum_spec.pro routine by summing over the pixels shown as a small yellow box in Fig. 8. The observations showed nonGaussian shaped line profiles. A narrow core and broad wings are clearly seen in the Si IV line profile. Such line profiles can be fitted with two Gaussian components. But in the case of other lines (O IV and S IV) in this window, the emission is very weak and it is difficult to fit two Gaussians. Therefore, in this analysis, 

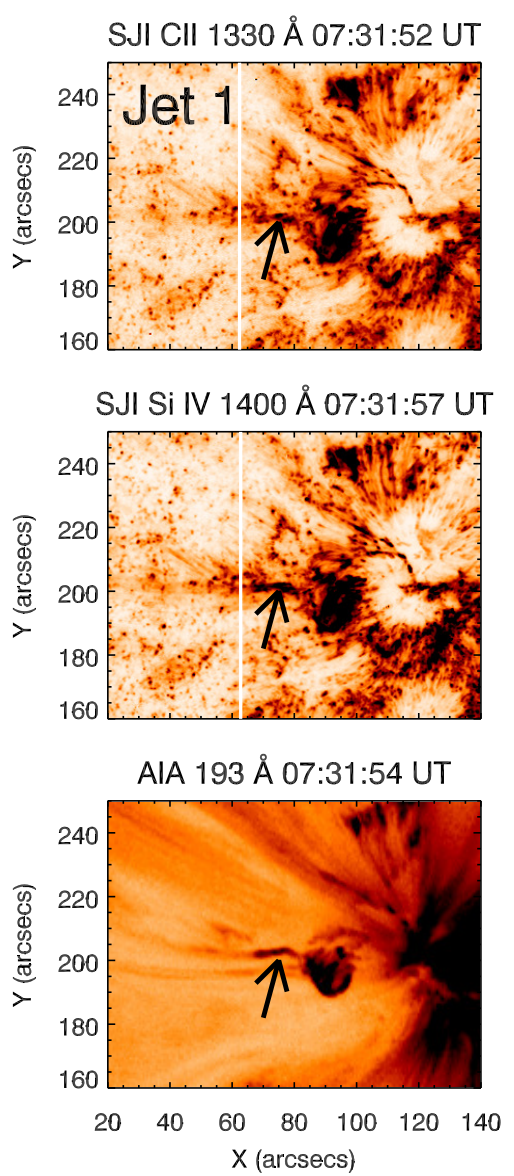
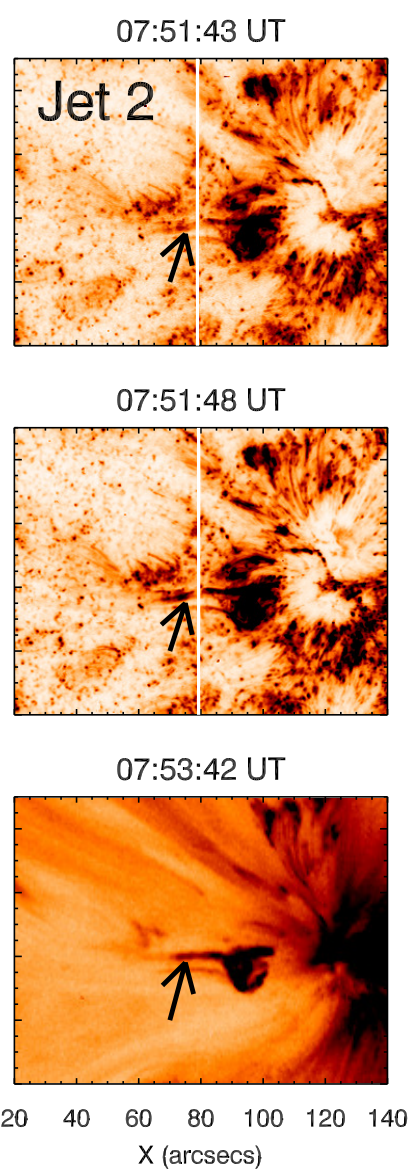

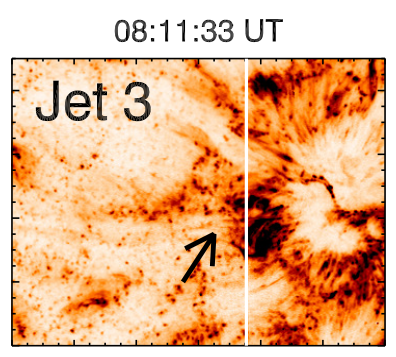

08:11:38 UT

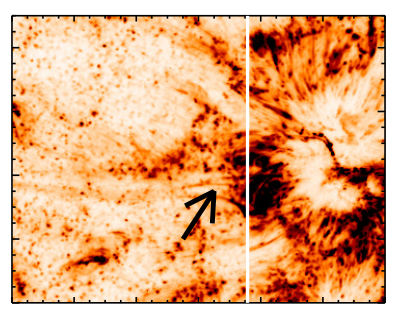

08:11:42 UT

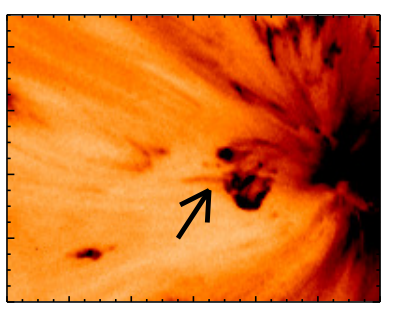

$20 \quad 40 \quad 60 \quad 80 \quad 100 \quad 120 \quad 140 \quad 20$ $\mathrm{X}(\operatorname{arcsecs})$
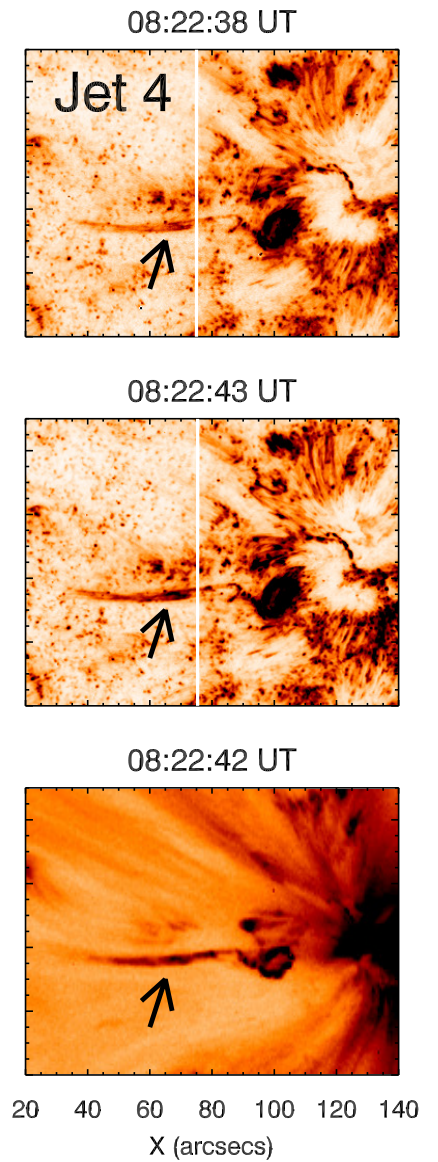

Fig. 3. Recurrent jets seen with AIA and IRIS SJI. The IRIS SJI C II $1330 \AA$ (top panel), Si IV $1400 \AA$ (middle panel), and the AIA $193 \AA$ (bottom panel) (reverse colour) images of four recurrent jets observed at 07:32 (jet 1), 07:53 (jet 2), 08:11 (jet 3) and 08:22 UT (jet 4) on July 10, 2015 originating from the penumbral region of the sunspot located at the eastern edge of an AR NOAA 12381 (N14 W25). The white overplotted line on the SJI images represents the spectrograph slit position (see movie) and black arrows indicate the spire structure of jets.

we used the intensities (in DN) calculated by summing the total intensity under the line profile and then converted them in the calibrated units of erg $\mathrm{cm}^{-2} \mathrm{~s}^{-1} \mathrm{sr}^{-1}$ at each pixel position using the iris_calib.pro routine and spectrograph effective areas (version 004) by considering a time-dependent degradation factor for the IRIS spectrograph. The blue vertical lines shown in Fig. 2 are the wavelength ranges over which we obtained the total counts for each line. We found good agreement in the counts obtained by fitting the Si IV line profile and the counts obtained by summing the total intensity under the line profile after subtracting a background. Taking account of spectral line shifts, we obtained intensities in the spire region by using a similar method over the wavelength ranges as shown in Fig. A.1.

\section{Data analysis and results}

\subsection{AIA-SJI-XRT co-alignment}

The AIA $1600 \AA$ channel observes emission from the C IV line and continuum emission from the chromosphere/transition region. The AIA 1600 and $1700 \AA$ images showed that the eastern part of the NOAA AR 12381 (N14 W25) was associated with a sunspot region. We used the sunspot's umbral-penumbral region and lightbridge region to precisely coalign all EUV/UV channels of AIA instrument. Also, the AIA $1600 \AA$ channel is the most suitable channel to coalign with the SJI images. We coaligned the SJI $1330 \AA$ channel which observes plasma at $T \sim 0.02 \mathrm{MK}$ emitted by the C II 1334/1335 A line with the AIA $1600 \AA$. Similarly, we coaligned SJI Si IV $1400 \AA$ channel with the AIA $1600 \AA$.

We followed the co-alignment procedure given in the Yoshimura \& McKenzie (2015) to carefully co-align XRT images with the AIA $335 \AA$ channel which is dominated by Fe XVI formed at $T=\sim 3 \mathrm{MK}$.

In this analysis, we are focusing on the C II 1334/1335 $\AA$ and Si IV $1400 \AA$ A windows observed by both IRIS instruments.

\subsection{Overview and kinematics}

The movie shows the evolution of the four recurrent jets in the SJI 1330, $1400 \AA$ and AIA $193 \AA$ channels during $\sim 1 \mathrm{~h}$ of observations from 07:29 to 08:35 UT. The jets originated from the eastern edge of the penumbral region of the sunspot. The multithermal AIA channels and both SJI channels showed the fine, multi-threaded structure of the jet-spire. Small plasma-blobs can also be seen moving along the spire structure (not shown in Fig. 3) in the movie (movie1.mp4) during 07:54:54-07:55:42 UT and 08:21:54-08:23:30 UT (time-stamp according to AIA $193 \AA$ A images).

Figure 3 shows frames from the movie in the SJI C II $1330 \AA$ (top panel), Si IV $1400 \AA$ (middle panel) passbands and the AIA $193 \AA$ (bottom panel) (reverse colour). The white overplotted line on the SJI images represents the spectrograph slit position and black arrows indicate the spire structure of jets. 
We measured the lifetime of the jets by tracking the jet's spire observed in the SJI Si IV $1400 \AA$ images. We labelled these jets as (the start and end times of jets are indicated in the parentheses): Jet 1 (07:29:22-07:32:59 UT), Jet 2 (07:48:58-07:58:30 UT), Jet 3 (08:11:08-08:17:50 UT), Jet 4 (08:19:07-08:30:27 UT).

Each jet is sampled in the different IRIS spectrograph raster scans. The four raster scans (the start and end times of each raster scan is indicated in the parentheses) - raster 1: (07:29:17-07:45:42 UT), raster 2: (07:45:4708:02:11 UT), raster 3: (08:02:17-08:18:41 UT) and raster 4: (08:18:46-08:35:11 UT). The spire and the footpoint region of four jets were sampled in each raster scan (see Fig. 6, top panel).

\subsection{Velocity calculation}

\subsubsection{Plane-of-sky velocities}

We calculated the plane-of-sky velocities of the jets in the lower corona using the AIA $171 \AA$ channel which is dominated by a single ion, i.e. Fe IX $(\log T[\mathrm{~K}]=5.85)$. The jet structures are clearly visible in this channel. We compared the velocities obtained in the lower corona with those obtained in the lower transition region using the SJI $1400 \AA$ channel.

Figures $4 \mathrm{a}$ and $5 \mathrm{a}$ show the SJI Si IV $1400 \AA$ and the AIA $171 \AA$ images respectively and the overplotted horizontal (green) and vertical (blue) lines represent two artificial slit positions where we obtained time-distance plots. Figures $4 \mathrm{~b}$ and $5 \mathrm{~b}$ show the time-distance plots for the vertical slit position. The features shown by blue arrows show the horizontal movement (from solar south to north) of the spire structure with time, representing the untwisting motion of the magnetic field lines associated with the jet spire structure (Shen et al. 2011; Hong et al. 2013; Schmieder et al. 2013; Panesar et al. 2016a). Figures 4c and 5c show the timedistance plots obtained from the horizontal slit position. Jet 1 and jet 3 were faint and their signatures were difficult to capture in the time-distance plot. Jet 2 and jet 4 are well observed in these plots and show multiple threads along the spire structure. Therefore, we only calculated the plane-of-sky velocities for jet 2 and jet 4 along five different threads in the AIA and SJI time-distance plots. The overplotted five long dashed green lines (named as S1, S2, S3, S4, and S5 respectively from left) represent threads along the jet 2 spire and dashed blue lines represent different threads along the jet 4 spire (named as S6, S7, S8, S9 and S10 respectively from left).

Table 2 provides details of the plane-of-sky velocities obtained from the AIA and SJI observations. In the time-distance plot obtained by using the SJI $1400 \AA$ images, we calculated average plane-of-sky velocities of $206 \pm 18 \mathrm{~km} \mathrm{~s}^{-1}$ for jet 2 along green long dashed lines. Similarly, we calculated velocities of $286 \pm 23 \mathrm{~km} \mathrm{~s}^{-1}$ for jet 4 along blue dashed lines. In the timedistance plot obtained by using the AIA $171 \AA$ images, we calculated average plane-of-sky velocities of $223 \pm 45 \mathrm{~km} \mathrm{~s}^{-1}$ for jet 2 along green long dashed lines. Similarly, we calculated velocities of $367 \pm 43 \mathrm{~km} \mathrm{~s}^{-1}$ for jet 4 along blue dashed lines. The averaged velocities for jet 4 were higher than those of jet 2 in both AIA and SJI observations. The averaged velocity of jet 4 was also higher in the AIA than in the SJI observations.

\subsubsection{Doppler velocity}

The IRIS spectrograph observes the strong neutral O I $1355.6 \AA$ line in the FUV short wavelength range. This line is formed at photospheric heights and generally shows Doppler shifts less (a) 10-Jul-2015 IRIS SJI Si IV $1400 \AA ̊$ 08:26:20 UT

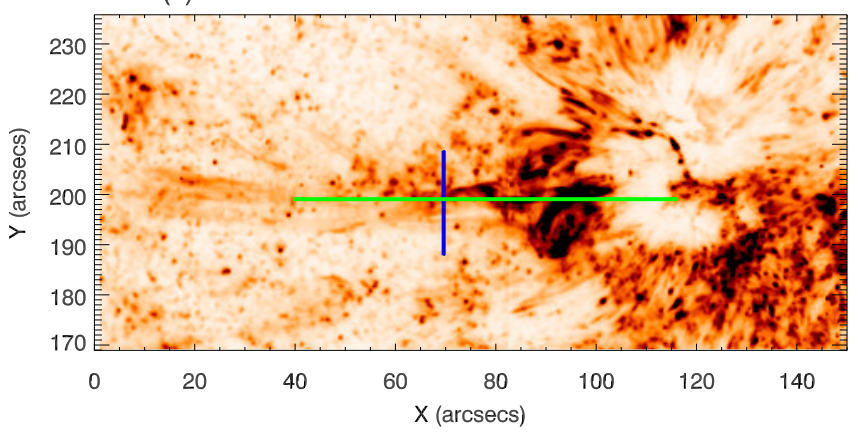

(b) time-distance plot (vertical slit)

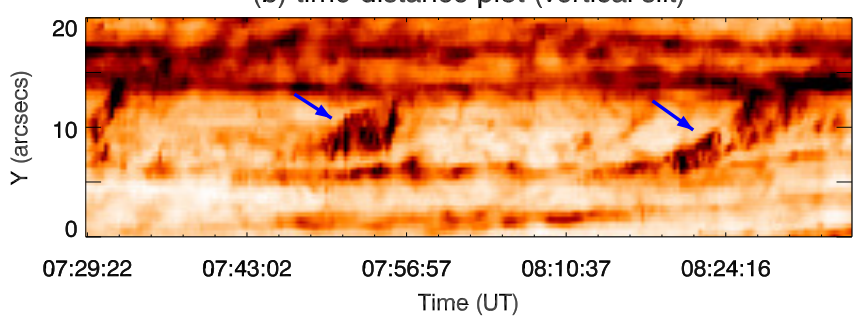

(c) time-distance plot (horizontal slit)

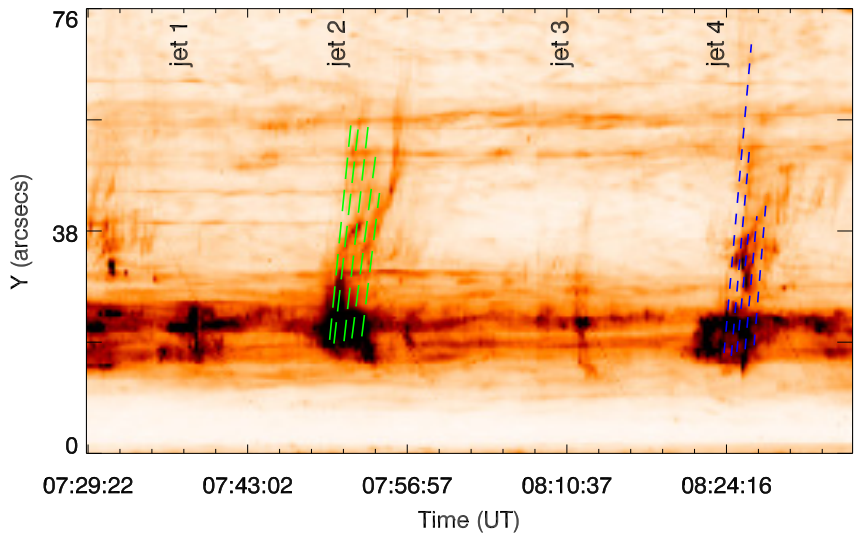

Fig. 4. SJI plane-of-sky velocities. Panel $a$ : the SJI Si IV $1400 \AA$ image (reverse colour) at 08:26:20 UT. The overplotted horizontal (green) and vertical (blue) lines represent two artificial slit positions where we obtained time-distance plots. Panel $b$ : the time-distance plot for the vertical slit position (reverse colour). The features shown by blue arrows represent an untwisting motion of the jet spire structures. Panel $c$ : the time-distance plot obtained from the horizontal slit position (reverse colour). The long dashed green lines are overplotted on threads of the spire of jet 2 and dashed blue lines are overplotted on threads of the spire of jet 4 . These lines were used to calculate the plane-of-sky velocities.

than $1 \mathrm{~km} \mathrm{~s}^{-1}$. So, it is the most suitable line for the wavelength calibration (Polito et al. 2015). We used this line for the absolute wavelength calibration for our data set (details are given in IRIS document ITN20).

Figure 6 shows four rasters observed during the recurrent activity of the jet. The start time for each raster is given in the $\mathrm{Si}$ IV intensity maps (reverse colour images in top panel). The signatures of the footpoint region are well observed in all rasters. The bright emission from the spire is nicely captured in jet 2 and jet 4 . Very faint emission is observed in case of jet 1 and jet 3 . The small overplotted green box indicates the region of the spire and yellow box indicates the foopoint region. The Doppler velocity maps (middle panel) shows blue shifted components associated with the spire structure and a red shifted component 
(a) 10-Jul-2015 AIA 171 Å 08:26:23 UT

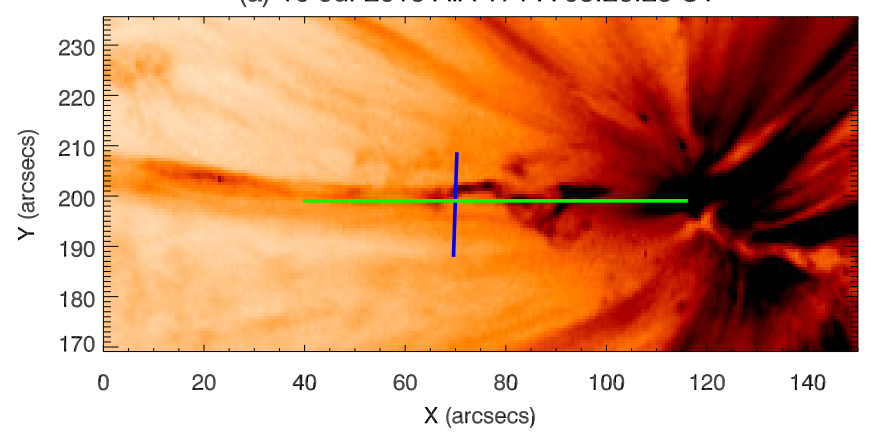

(b) time-distance plot (vertical slit)

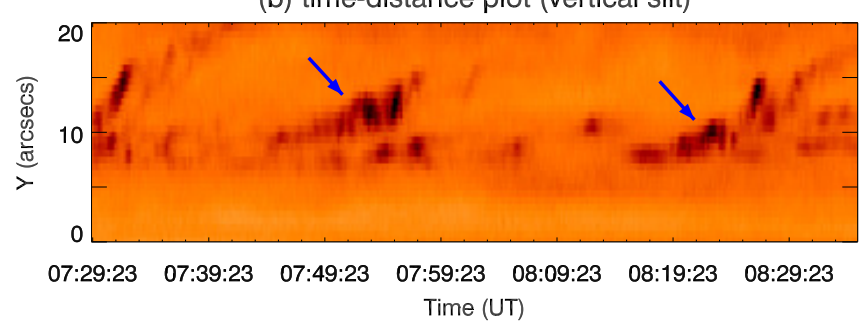

(c) time-distance plot (horizontal slit)

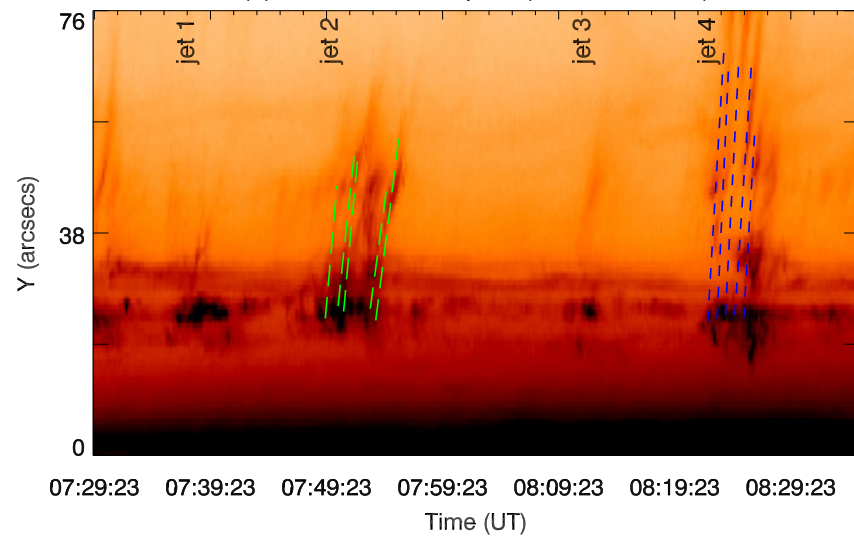

Fig. 5. AIA plane-of-sky velocities. Panel $a$ : the AIA $171 \AA$ image (reverse colour) at 08:26:23 UT and the overplotted horizontal (green) and vertical (blue) lines represent two artificial slit positions where we obtained time-distance plots. Panel $b$ : the time-distance plot for the vertical slit position (reverse colour). The features shown by blue arrows represent an untwisting motion of the jet spire structures. Panel $c$ : the time-distance plot obtained from the horizontal slit position (reverse colour). The long dashed green lines are overplotted on threads of the spire of jet 2 and dashed blue lines are overplotted on threads of the spire of jet 4 . These lines were used to calculate the plane-of-sky velocities.

in the footpoint structure for all jets. We calculated the Doppler velocities in these regions (shown by black arrows) for four jets (see Table 2). The Doppler velocity in the spire region of the jet 4 $\left(32 \mathrm{~km} \mathrm{~s}^{-1}\right)$ was found to be higher than the other three jets. In the footpoint region, the Doppler velocities for all jets were very similar around $11 \mathrm{~km} \mathrm{~s}^{-1}$. The Doppler velocities obtained here were found to be smaller compared with the results obtained by Cheung et al. (2015) and Kim et al. (2007)

\subsection{Nonthermal width}

The nonthermal width in the region of the spire and footpoint (same boxed region where we calculated the Doppler velocities) was calculated using the iris_nonthermalwidth.pro routine available in the SSW, assuming an instrumental full-width-halfmaximum (FWHM) of $0.0318 \AA$ for the FUV long wavelength range. The Si IV $1402.77 \AA$ line was fitted with a single Gaussian component, which is a good approximation for the core of the line.

Figure 6 (bottom panel) shows the nonthermal velocity maps for the four jets. The small overplotted green box (shown by white arrows) indicates the region of the spire and the yellow box (shown by white arrows) indicates the foopoint region where we calculated nonthermal velocities (refer Table 2). The nonthermal velocities were found to be higher in the spire regions for all jets than in the foopoint regions. There are some regions in the footpoint where we found very high nonthermal velocities (e.g. the loop-like structure seen in the footpoint region of jet 1 near to yellow box). The nonthermal velocities obtained here (refer Table 2) were found to be smaller than the results obtained by Kim et al. (2007).

\subsection{O IV (1399.8/1401.2 Å) density diagnostic}

The IRIS spectrograph provides an opportunity to perform density diagnostics using the O IV intercombination lines observed between 1397 and $1407 \AA$ wavelength range (Polito et al. 2016). We used the ratio of intensities for the O IV $(\lambda 1399.8 / \lambda 1401.2)$ density-sensitive pair of lines to derive electron number densities in the region of the spire and footpoint of the jet 4 . We used the CHIANTI atomic database v.8 (Dere et al. 1997; Del Zanna et al. 2015) in this analysis and assumed a temperature of maximum ionization of $\log T[\mathrm{~K}]=5.15$. Figure 7 shows the theoretical intensity ratio for the O IV $(\lambda 1399.8 / \lambda 1401.2)$ line pair as a function of electron number density. The blue and red triangles represent the values measured for the spire $\left(N_{\mathrm{e}}=2.0 \times 10^{10} \mathrm{~cm}^{-3}\right)$ and footpoint $\left(N_{\mathrm{e}}=7.6 \times 10^{10} \mathrm{~cm}^{-3}\right)$ of jet 4 . The errors were calculated by considering a $20 \%$ uncertainty. The electron densities were found to be slightly higher in the footpoint region than in the spire.

\subsection{DEM analysis}

In our previous study of jets (Mulay et al. 2016), we obtained DEMs using only AIA data. This gave good results in the temperature range 1-3 MK. The main aim here is to combine IRIS and AIA observations to get a better constraint on the lower temperatures $(\log T[\mathrm{~K}]<5.4)$ in the DEM analysis. Also, we wanted to check whether emission at temperatures $T>2 \mathrm{MK}$ is present or not, by estimating the Fe XVIII emission from the AIA $94 \AA$ channel and comparing that with XRT temperatures obtained from the filter-ratio method.

The DEM analysis was performed by combining spectra from the IRIS spectrograph Si IV $1402.77 \AA(\log T[\mathrm{~K}]=4.9)$ and O IV $1401.16 \AA(\log T[\mathrm{~K}]=5.15)$ lines and images from multithermal AIA channels $(94,131,171,193,211$ and $335 \AA$ ) which are sensitive to coronal temperatures. In this section, we describe the coalignment procedure used for the AIA images and IRIS rasters to prepare input data for a DEM analysis and we then show DEM results for jet 4.

\subsubsection{DEM input}

A direct comparison of the IRIS spectrograph rasters and the AIA images is not straightforward for the following reasons: 1) the AIA channels are sensitive to multiple temperatures whereas the IRIS spectrograph is a spectrograph, and hence sensitive to a very 

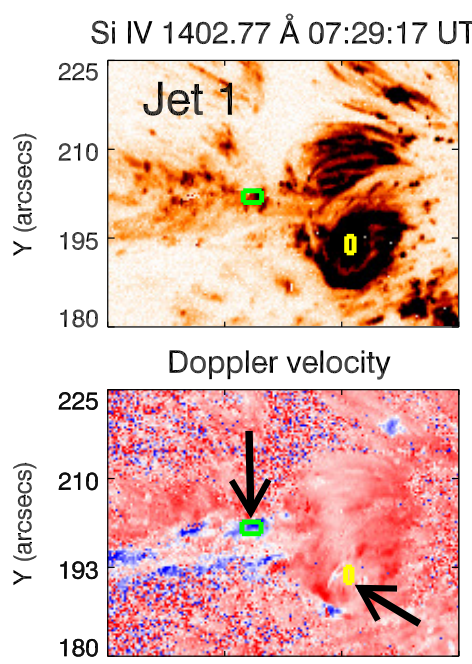

Nonthermal width

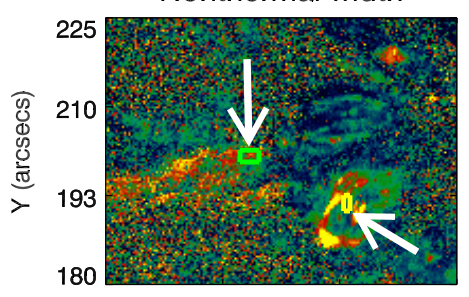

53
07:45:47 UT
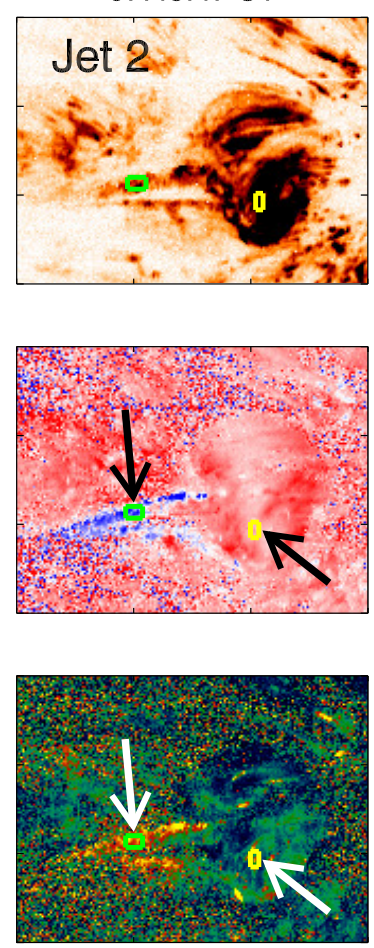

93

$\mathrm{X}(\operatorname{arcsecs})$

08:02:17 UT
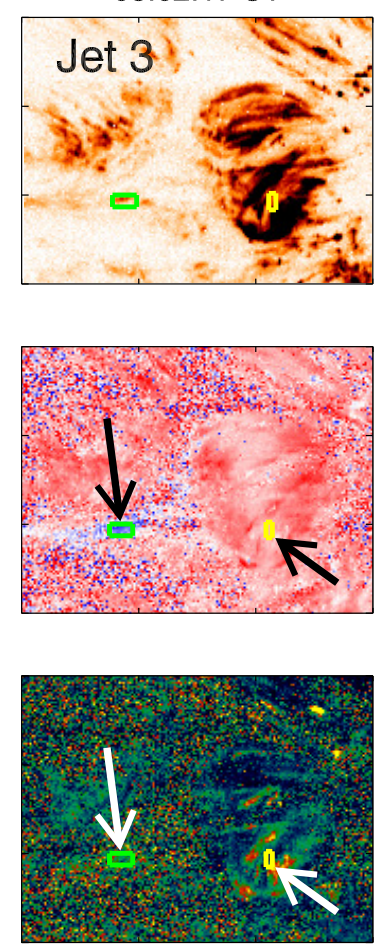

$76 \quad 96 \quad 11560$
08:18:46 UT
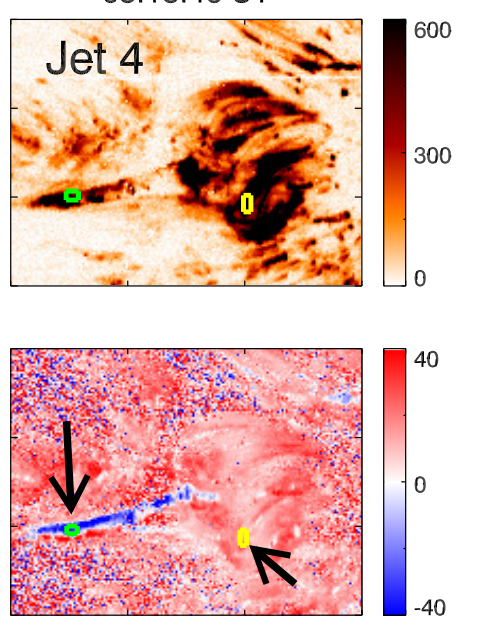

$\frac{\pi}{\frac{\sigma}{E}}$

Fig. 6. IRIS spectrograph images of the recurrent jet. The Si IV intensity maps (top panel; reverse colour), Doppler velocity maps (middle panel) and nonthermal velocity maps (bottom panel) for four rasters. The times indicate the start time for each raster. The oveplotted green and yellow boxes show the spire and footpoint regions respectively. These regions were used for the calculation of Doppler (shown by black arrows) and nonthermal (shown by white arrows) velocities.

Table 2. Velocities of recurrent jets for the spire and footpoint region (in parentheses).

\begin{tabular}{lcccc}
\hline \hline $\begin{array}{l}\text { Velocity } \\
\left(\mathrm{km} \mathrm{s}^{-1}\right)\end{array}$ & Jet 1 & $\begin{array}{c}\text { Jet 2 } \\
\text { (green) }\end{array}$ & Jet 3 & $\begin{array}{c}\text { Jet 4 } \\
\text { (blue) }\end{array}$ \\
\hline Plane-of-sky (aia) & - & $271(\mathrm{~S} 1)$ & - & 371 (S6) \\
Plane-of-sky (aia) & - & $217(\mathrm{~S} 2)$ & - & 332 (S7) \\
Plane-of-sky (aia) & - & $268(\mathrm{~S} 3)$ & - & 429 (S8) \\
Plane-of-sky (aia) & - & $172(\mathrm{~S} 4)$ & - & 321 (S9) \\
Plane-of-sky (aia) & - & $188(\mathrm{~S} 5)$ & - & $382(\mathrm{~S} 10)$ \\
\hline Average plane-of-sky (aia) & - & $223 \pm 45$ & - & $366 \pm 43$ \\
\hline Plane-of-sky (sji) & - & $223(\mathrm{~S} 1)$ & - & $272(\mathrm{~S} 6)$ \\
Plane-of-sky (sji) & - & $218(\mathrm{~S} 2)$ & - & $280(\mathrm{~S} 7)$ \\
Plane-of-sky (sji) & - & $216(\mathrm{~S} 3)$ & - & $321(\mathrm{~S} 8)$ \\
Plane-of-sky (sji) & - & $185(\mathrm{~S} 4)$ & - & 263 (S9) \\
Plane-of-sky (sji) & - & $189(\mathrm{~S} 5)$ & - & $298(\mathrm{~S} 10)$ \\
\hline Average plane-of-sky (sji) & - & $206 \pm 18$ & - & $286 \pm 23$ \\
\hline Doppler (Si IV) & $-22 \pm 18(10 \pm 3)$ & $-16 \pm 13(11 \pm 2)$ & $-16 \pm 13(11 \pm 2)$ & $-32 \pm 6(13 \pm 4)$ \\
Nonthermal (Si IV) & $54 \pm 12(47 \pm 18)$ & $72 \pm 14(48 \pm 14)$ & $47 \pm 14(39 \pm 20)$ & $69 \pm 6(53 \pm 14)$ \\
\hline
\end{tabular}

Notes. See text for detailed discussion. S1-5: green long dashed lines along different threads of the spire of jet 2. S6-10: blue dashed lines along different threads of the spire of jet 4 (see Figs. $4 \mathrm{c}$ and $5 \mathrm{c}$ ), the numbers in parentheses represent velocities calculated in the footpoint region.

narrow temperature range; the slit on the spectrograph rasters over the region on the solar surface and creates monochromatic images; 2) the temporal and spatial resolution for the AIA and IRIS instruments are different and the exposure times also differ.

We adopted a method described in Del Zanna et al. (2011) to coalign these datasets. Firstly, we rebinned the calibrated C II, Si IV and O IV images (in erg $\mathrm{cm}^{-2} \mathrm{~s}^{-1} \mathrm{sr}^{-1}$ units) to the AIA pixel resolution $\left(0.6^{\prime \prime}\right)$. Then we took an average over the AIA $1600 \AA$ images which is close in time with each IRIS raster position. We created a new time-averaged AIA $1600 \AA$ image by combining each averaged slice obtained from the method. We compared the new time-averaged AIA $1600 \AA$ image with the C II calibrated rebinned raster image and precisely coaligned them by comparing the sunspot penumbral and light-bridge regions which have distinctive features.

We followed a similar procedure to obtain time-averaged images for each AIA channel and used them as an input in the DEM analysis. Figure 8 shows the time-averaged (reverse colour) 


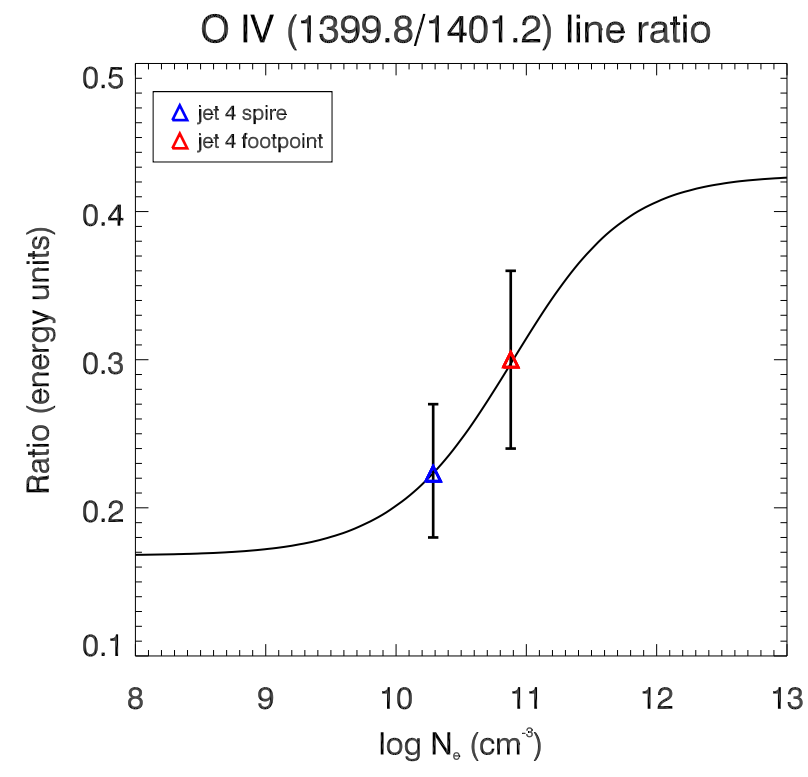

Fig. 7. O IV density measurements. The theoretical intensity ratio for the O IV ( $\lambda 1399.8 / \lambda 1401.2 \AA)$ line pair as a function of electron density. The blue and red triangles represent observed densities for the spire $\left(N_{\mathrm{e}}=2.0 \times 10^{10} \mathrm{~cm}^{-3}\right)$ and footpoint $\left(N_{\mathrm{e}}=7.6 \times 10^{10} \mathrm{~cm}^{-3}\right)$ of the jet 4 . The black vertical lines represent error bars calculated by considering $20 \%$ uncertainty.

AIA images and rebinned calibrated C II, Si IV and O IV images obtained for the jet 4 . The green and yellow oveplotted boxes represent the region of the spire and footpoint structure respectively. The foreground emission was significant in the AIA 171, 193 and $211 \AA$ channels (see Cols. 2 and 7 in Table 4). We subtracted this emission in all AIA channels by taking averaged intensities in the boxed regions during the pre-jet phase at 08:10:35 UT (see Fig. A.2). The obtained intensities were then used as an input to calculate DEMs (see Cols. 4 and 9 in Table 4).

In this analysis, we used the CHIANTI atomic database v. 8 (Dere et al. 1997; Del Zanna et al. 2015), the photospheric abundances by Asplund et al. (2009) and the electron number densities obtained in the same regions of spire and the footpoint (see Sect. 3.5). The temperature responses for the AIA channels were calculated using the procedure described in Del Zanna et al. (2011). The effective areas for each AIA channel were calculated using the aia_get_response . pro routine by considering a time-dependent degradation factor for each channel.

The contribution function for the Si IV $1402.77 \AA$ and O IV 1401.16 lines were calculated using the gofnt.pro routine using electron number densities obtained in the region of the spire and footpoint of jet 4 (see Sect. 3.5).

\subsubsection{DEM results}

The DEM curves were calculated over the region of the spire and footpoint structure (see green and yellow small boxes in Fig. 8) in the temperature interval $4.1<\log T[\mathrm{~K}]<7.0$ using the DEM inversion xrt_dem_iterative2.pro method (Weber et al. 2004) available in the SSW. We modified this routine substantially in order to combine the IRIS spectra and the AIA observations in the DEM analysis (see details in Appendix A). In the spire structure, the green box covers seven IRIS spectrograph slit positions (08:20:29-08:21:00 UT) and the yellow box covers four IRIS spectrograph slit positions (08:28:03-08:28:18 UT) over the region of the footpoint. The times indicated in parenthesis are the start times for the first and last slit exposure corresponding to the boxed regions.

Figure 9 shows the DEM curves for the spire (top panel) and the footpoint (bottom panel) of jet 4 . We obtained estimates of uncertainties on the best-fit DEM curves by randomly varying the input intensities by $20 \%$. We performed 300 Monte Carlo (MC) simulations on the intensities. In each temperature bin, the errors are plotted with different colour bars. $50 \%$ of solutions closest to best-fit DEM are shown by blue, $80 \%$ by red and $95 \%$ by yellow. Table 3 provides the details of the plasma parameters calculated for jet 4 .

Table 4 (Cols. 3 and 8) shows the observed intensities (DN/s) in each AIA channel obtained from Fig. 8. Columns 4 and 9 indicate the AIA intensities (DN/s) obtained after subtraction of pre-jet intensities from the observed intensities. The intensities obtained from the Si IV and O IV raster images are in erg $\mathrm{cm}^{-2} \mathrm{~s}^{-1} \mathrm{sr}^{-1}$ units. The predicted intensities (Cols. 5 and 10) were calculated from the DEM curves. The ratio of observed to predicted intensities is given in the parentheses.

In the DEM analysis, significant cool emission was found in the spire and the footpoint region (see Fig. 9). The hot emission was peaked at $\log T[\mathrm{~K}]=6.5$ in the footpoint region. The spire DEM shows a plateau in the temperature range from $\log T[\mathrm{~K}]=5.6$ to 5.9. Using the IRIS observations, both DEM curves are very well constrained in the lower temperature range $(\log T[\mathrm{~K}]<5.4)$ and the predicted intensities in the Si IV, O IV lines are in good agreement with the observed ones (see Table 4, Cols. 5 and 10). For the temperatures $\log T[\mathrm{~K}]>6.1$, the spire DEM curve falls sharply. This indicates that the main coronal contribution is from the lower temperature plasma ( $\sim 1 \mathrm{MK})$. In the case of the footpoint region, the DEM shows the presence of higher temperature plasma $(\log T[\mathrm{~K}]>6.3)$ along with emission from plasma at lower temperatures.

We performed a DEM analysis for jet 4 (same method as above) using only the AIA observations to see the differences in the temperature and DEM curves. The DEM curves were calculated over the same region of the spire and footpoint structure (see green and yellow small boxes in Fig. 8). Since the AIA channels are not sensitive to temperatures $\log T[\mathrm{~K}]<5.2$, the DEMs were calculated in the temperature interval $5.2<\log T[\mathrm{~K}]<7.0$. We used the same input data for the DEM analysis as given in Table 4 (Cols. 4 and 9.)

Figure 10 shows the DEM curves for the spire (top panel) and the footpoint (bottom panel) of jet 4 obtained using only AIA observations. By considering a $20 \%$ uncertainty, we obtained error bars on the best-fit DEM curves by performing $300 \mathrm{MC}$ simulations on the intensities. In each temperature bin, the errors are plotted with different colour bars. $50 \%$ of solutions closest to the DEM best-fit are shown by blue, $80 \%$ by red and $95 \%$ by yellow. Table 4 (Cols. 6 and 11) indicate the predicted intensities (DN/s) obtained from the DEM analysis for jet 4 using only AIA data. The predicted intensities show very good agreement with the observed ones.

In the spire region, the DEM peaks at $\log T[\mathrm{~K}]=5.7$ and shows another peak at high temperatures at $\log T[\mathrm{~K}]=6.5$. The DEM falls sharply for the temperatures below $\log T[\mathrm{~K}]=5.6$ and above $\log T[\mathrm{~K}]=6.5$. The error bars are quite large over the entire temperature interval. In the footpoint region, the DEM peaks at $\log T[\mathrm{~K}]=6.5$ and falls sharply at higher temperatures. The large error bars for the temperatures $\log T[\mathrm{~K}]<6.3$ shows the uncertainties in the DEM values.

By the inclusion of the IRIS spectra in the DEM analysis, the lower temperatures $\log T[\mathrm{~K}]<5.4$ are very well constrained and DEM curves give a clear indication of low temperature present 


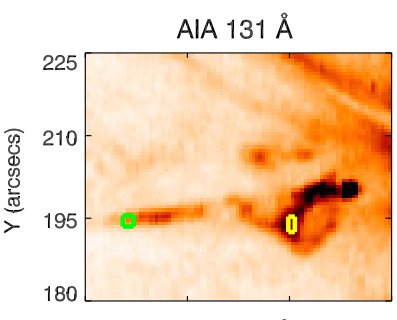

AIA $335 \AA$

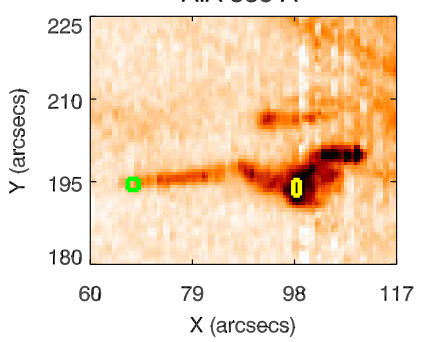

AIA $171 \AA$

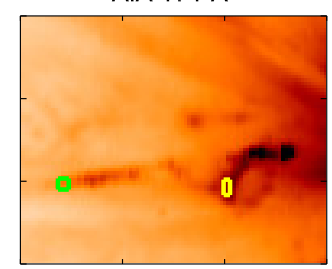

Si IV $1402.77 \AA$

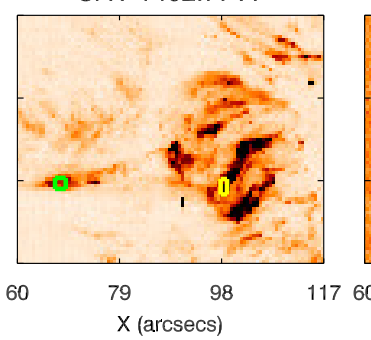

AIA $193 \AA$

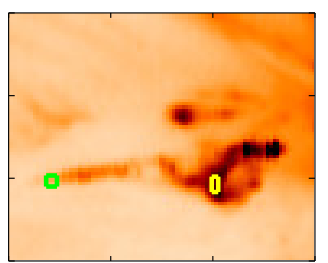

O IV $1401.16 \AA$

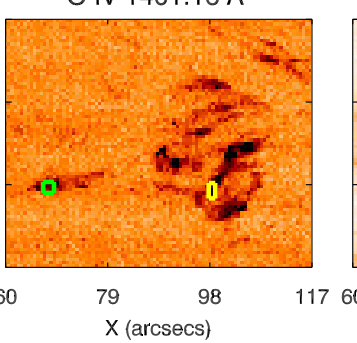

AIA $211 \AA$

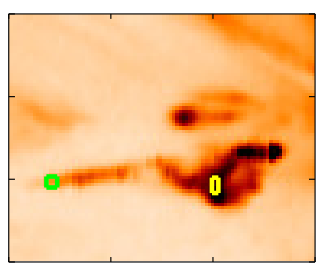

C II $1334.43 \AA$

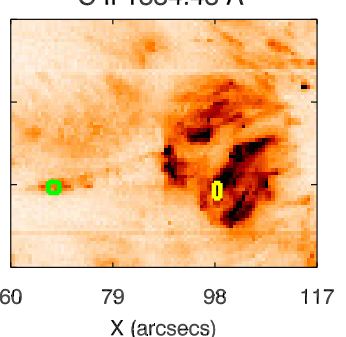

AIA $94 \AA$

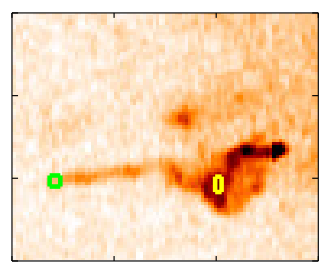

Fe XVIII $93.932 \AA$

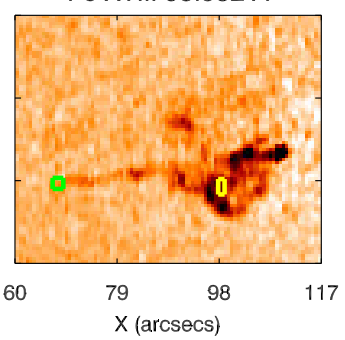

Fig. 8. AIA and IRIS images indicating regions for DEM determination. The time-averaged (reverse colour) AIA and Fe XVIII images together with rebinned, calibrated Si IV, O IV, C II raster images obtained for jet 4 . The averaged intensities in the green and yellow oveplotted boxes were used to calculate DEMs for the spire (08:20:29-08:21:00 UT) and footpoint (08:28:03-08:28:18 UT) structures respectively. The times indicated in parentheses are the start times for the first and last slit exposures corresponding to the boxed regions.
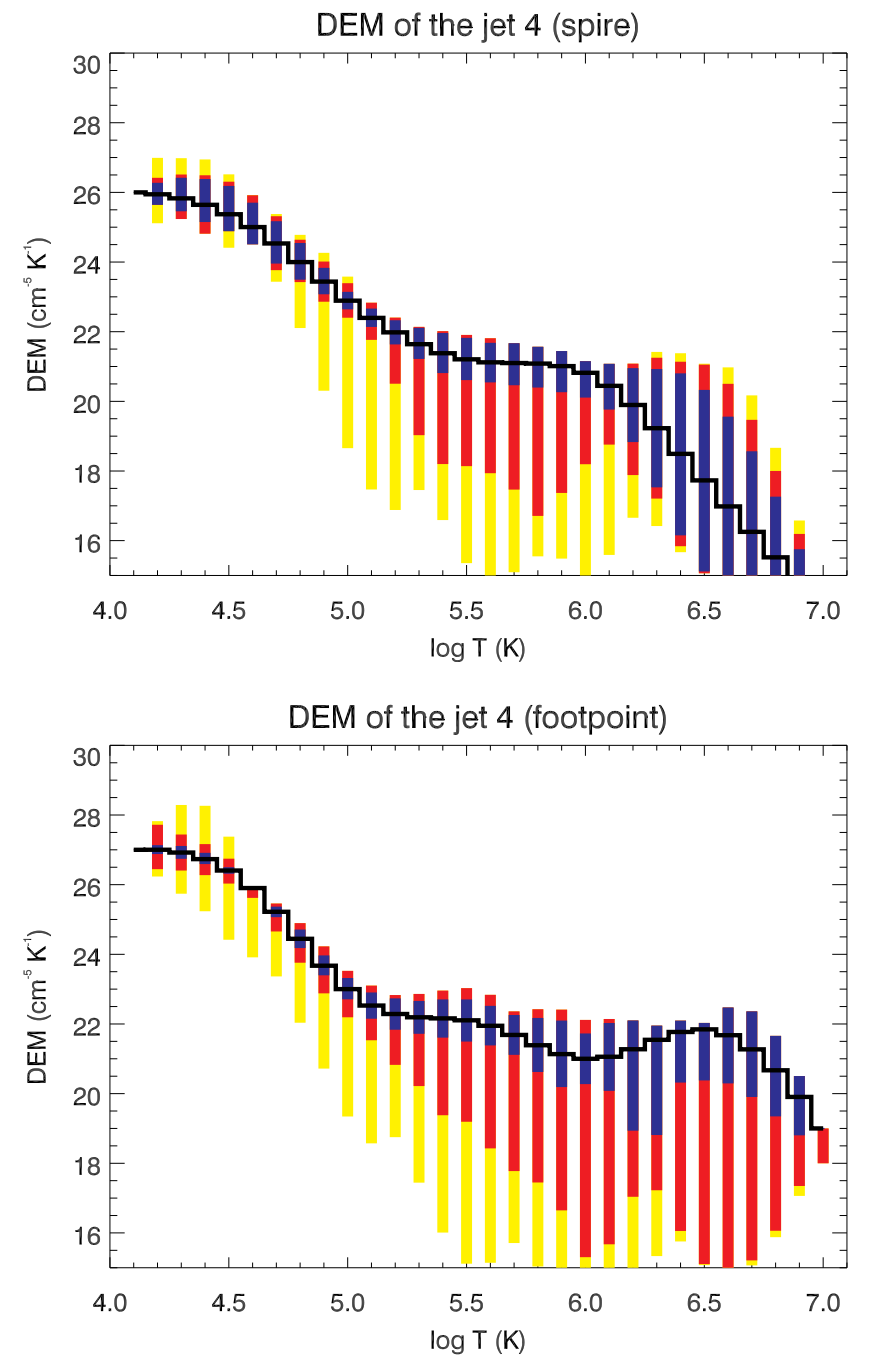

Fig. 9. DEM results for spire. The DEM curves for the spire (top panel) and footpoint (bottom panel) region of the jet 4 . The overplotted colour bars show $50 \%$ (blue), $80 \%$ (red) and $95 \%$ (yellow) of the $300 \mathrm{MC}$ solutions relative to the best-fit DEM.
Table 3. Plasma parameters for the spire and footpoint of the jet 4 .

\begin{tabular}{lcc}
\hline \hline Plasma parameters & Jet-spire & Jet-footpoint \\
\hline & (using AIA and IRIS) \\
First slit time (UT) & $08: 20: 29$ & $08: 28: 03$ \\
Last slit time (UT) & $08: 21: 00$ & $08: 28: 18$ \\
$\log T(\mathrm{~K})$ for peak DEM & $5.6-5.9$ & 6.5 \\
Peak DEM $\left(\mathrm{cm}^{-5} \mathrm{~K}^{-1}\right)$ & $1.3 \times 10^{21}$ & $7 \times 10^{21}$ \\
Electron density from O IV $\left(\mathrm{cm}^{-3}\right)$ & $2.0 \times 10^{10}$ & $7.6 \times 10^{10}$ \\
Doppler velocity $\left(\mathrm{km} \mathrm{s}^{-1}\right)$ & -32.0 & 13.4 \\
Total EM $\left(\mathrm{cm}^{-5}\right)(4.1<\log T<7.0)$ & $9.7 \times 10^{30}$ & $9.7 \times 10^{31}$ \\
Electron pressure $\left(P=N_{\mathrm{e}} T_{\mathrm{e}}\right)\left(\mathrm{cm}^{-3} \mathrm{~K}\right)$ & $2.8 \times 10^{15}$ & $1.1 \times 10^{16}$ \\
\hline \multicolumn{3}{c}{$(\mathrm{using}$ AIA only) } \\
log $T(\mathrm{~K})$ for peak DEM & 5.7 \\
Peak DEM $\left(\mathrm{cm}^{-5} \mathrm{~K}^{-1}\right)$ & $1.6 \times 10^{21}$ & $1.1 \times 10^{22}$ \\
Total EM $\left(\mathrm{cm}^{-5}\right)(5.2<\log T<7.0)$ & $4.5 \times 10^{27}$ & $3.1 \times 10^{28}$ \\
\hline
\end{tabular}

Notes. The time indicates the start times for the first and last slit exposures corresponding to the box regions for which we calculated DEMs in the region of the spire (green box) and the footpoint (yellow box; Fig. 8) of jet 4 (for details see Sect. 3.6.2). $T_{\mathrm{e}}=10^{5.15} \mathrm{~K}$ for O IV.

both in the spire and the footpoint regions along with some coronal emission. We calculated the total emission measure (EM) in the spire and footpoint structures in the temperature range $4.1<\log T[\mathrm{~K}]<7.0$ using the AIA and IRIS DEM. The EM for the footpoint region is one order of magnitude higher than in the spire. We also calculated the EM in both regions using only AIA images in the temperature range $5.2<\log T[\mathrm{~K}]<7.0$ and found that the values are almost three orders of magnitude lower (see Table 3).

\subsection{Filling factor in the transition region}

We calculated the filling factor $f=E M /\left(N_{\mathrm{e}}^{2} h\right)$ for the spire using the O IV electron density. O IV has a relatively broad contribution function $G(T)$ with a temperature of maximum at $\log T[\mathrm{~K}]=5.15$. We took the temperature range $4.9<\log T<5.4$ for the EM calculation, which is $1 / 10$ th of the peak of the $G(T) \times$ DEM, obtaining $E M=5.3 \times 10^{27} \mathrm{~cm}^{-5}$. The 

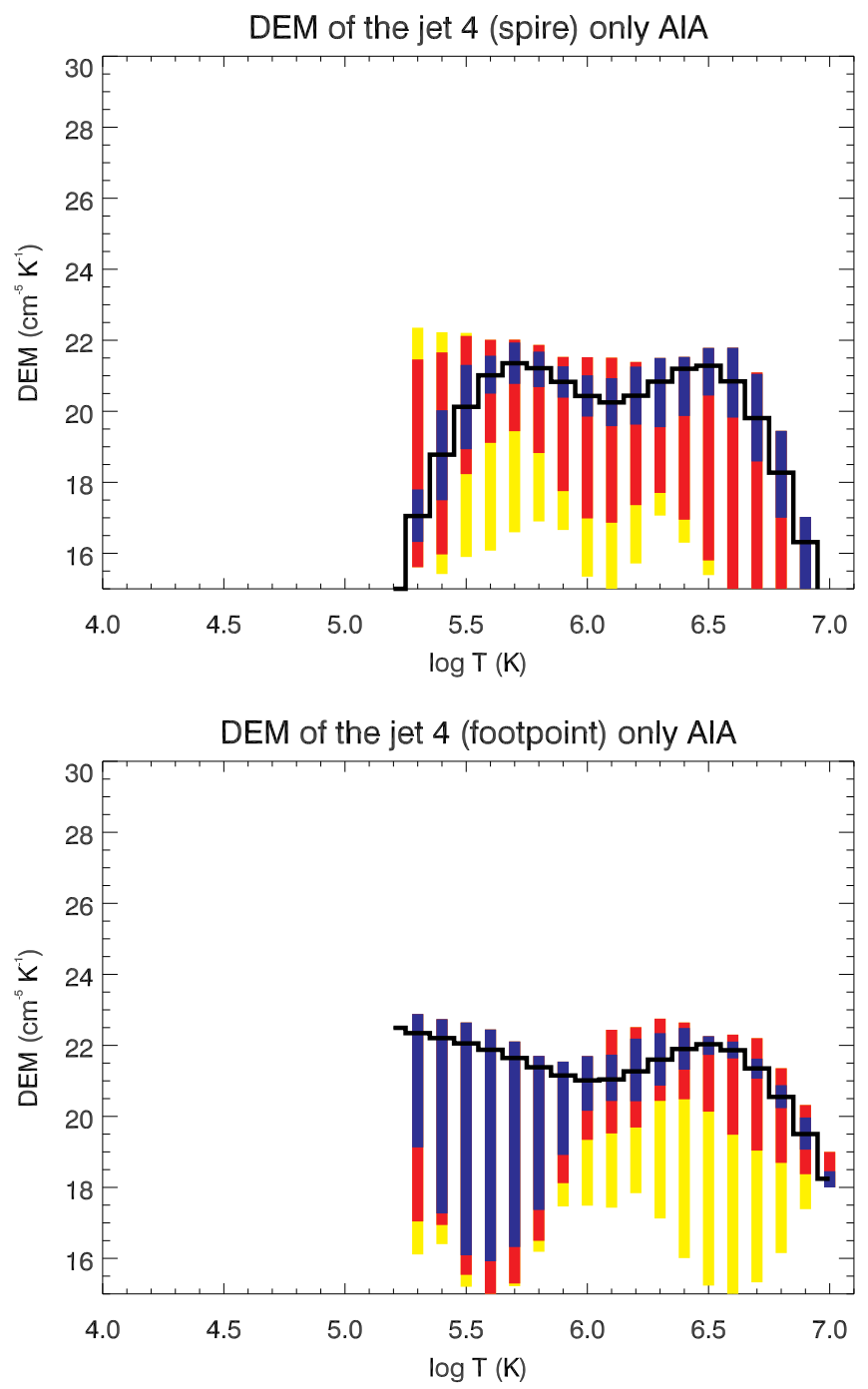

Fig. 10. DEM results for footpoint. The DEM curves for the spire (top panel) and footpoint (bottom panel) region of the jet 4 using only AIA images shown in Fig. 8. The overplotted colour bars show 50\% (blue), $80 \%$ (red) and $95 \%$ (yellow) of the $300 \mathrm{MC}$ solutions relative to the best-fit DEM.

width of the spire $h$ was estimated to be $\sim 2^{\prime \prime}$ and we obtained a filling factor of 0.1 for the spire at this temperature.

\subsection{Fe XVIII (93.932 $\AA$ ) emission in the jet footpoint}

Assessing the presence of hot plasma $(>2 \mathrm{MK})$ within a jet is important, because it is predicted by some theoretical models/simulations. We investigated further the reliability of the hot plasma ( $>2 \mathrm{MK}$ ) deduced from the DEM curves. We estimated the Fe XVIII (93.932 ̊) count rates within the AIA $94 \AA$ channel using a semi-empirical method, a combination of the AIA 211 and $171 \AA$ channels (Del Zanna 2013). We followed a similar coalignment procedure as that described in Sect. 3.6.1 to create a Fe XVIII map. Figure 8 (bottom panel, Col. 5) shows the Fe XVIII map for the jet 4 (reverse colour). The overplotted green and yellow boxes show the region of the spire and footpoint respectively. There is a clear indication of weak Fe XVIII emission in the footpoint region (2.1 DN/s, see Table 4, Col. 10). The estimated Fe XVIII count rates within the AIA $94 \AA$ channel using the DEM are 2.4, i.e. in very good agreement with those estimated using the semi-empirical method. In the spire, the Fe XVIII emission is very faint and not detectable.

Even though we see Fe XVIII emission in the jet footpoint region, it does not mean that the plasma temperature is actually $\log T[\mathrm{~K}]=6.85$. In fact, the contribution function for Fe XVIII is broad, ranging from $\log T[\mathrm{~K}]=6.25$ to 7.38 , with a maximum at $\log T[\mathrm{~K}]=6.85$. In order to investigate where the line is formed, we calculated its effective temperature, i.e. the DEM-weighted average temperature $T=\int \operatorname{DEM}(T) \times$ $T \mathrm{~d} T / \int \operatorname{DEM}(T) \mathrm{d} T$ of the footpoint region in the temperature range between $\log T[\mathrm{~K}]=6.2$ and 7.0. We found the effective temperature to be $\log T[\mathrm{~K}]=6.5$. This is in agreement with the average temperature obtained from the XRT images (Sect. 3.9) and the peak shown in the DEM for the footpoint region (Fig. 9, lower panel).

\subsection{Temperature analysis with XRT filters}

The XRT instrument recorded activity for the first 2 jets during 07:30-08:00 UT and at the later phase of jet 4 at 08:34 UT. Nearsimultaneous observations in different XRT filters have given us an opportunity to estimate average temperatures in jets using the filter-ratio method (for details see Sect. 5.1 of Narukage et al. 2011).

We used the make_xrt_wave_resp.pro routine to calculate the effective areas and spectral responses for the Al-poly and Be-thin filters. The temperature responses for these filters (see Fig. A.3) were obtained using the procedure given in Del Zanna et al. (2011). In this analysis, we used CHIANTI v.8 (Del Zanna et al. 2015) atomic database, an electron number density of $N_{\mathrm{e}}=1 \times 10^{10} \mathrm{~cm}^{-3}$ and the photospheric abundances of Asplund et al. (2009).

Figure 11 shows the XRT Al-poly (top panel), Be-thin (middle panel) images (reverse colour) and XRT temperature maps (bottom panel) during the period when jet 1 and 2 occurred. All the images are de-rotated to 07:29 UT. The temperature of the jet footpoint did not change dramatically over the period of time. A faint spire structure was observed in the XRT images indicative of a temperature around $\log T[\mathrm{~K}]=6.5$ at $07: 31 \mathrm{UT}$. The small loop like structure observed in the footpoint region is the same as that observed in Fig. 12 at 07:39 UT (shown by black arrows.) In most places in the footpoint region, the temperature was found to be $\log T[\mathrm{~K}]=6.5$ but in few places it rose up to $\log T[\mathrm{~K}]=6.6-6.7$. These observations confirm the multithermal and hot nature of the jet footpoint.

\subsection{Cool and hot temperature components in the jet footpoint from IRIS and AIA}

The temporal evolution of the cool and hot emission in the footpoint region has been investigated using near simultaneous observations from the SJI (17 s) and the AIA (12 s) channels over the period of recurrent jet activity. We used the SJI Si IV $1400 \AA$, C II $1330 \AA$ images to compare the low temperature plasma formed at $\log T[\mathrm{~K}]=4.9-5.1$ with hot emission observed in the Fe XVIII images obtained from the AIA $94 \AA$ channel. We carefully coaligned these images to check the spatial correlation between hot and cool components seen in the footpoint structure.

Figure 12 shows the SJI C II $1330 \AA$ (top panel), Si IV $1400 \AA$ (middle panel) and the Fe XVIII images (bottom panel; reverse colour) during one hour period of the recurrent jet activity. All the images are de-rotated to 07:29 UT. A small loop-like structure associated with the 
S. M. Mulay et al.: Cool and hot emission in a recurring active region jet

Table 4. Observed and predicted AIA and IRIS spectrograph intensities obtained from the DEM analysis for the spire and footpoint of the jet 4.

\begin{tabular}{|c|c|c|c|c|c|c|c|c|c|c|}
\hline (Col. 1) & (Col. 2) & (Col. 3) & (Col. 4) & (Col. 5) & (Col. 6) & (Col. 7) & (Col. 8) & (Col. 9) & (Col. 10) & (Col. 11) \\
\hline $\begin{array}{l}\text { Band } \\
(\AA)\end{array}$ & $\begin{array}{l}\text { Pre-jet } \\
\text { (spire) }\end{array}$ & $\begin{array}{l}\text { Observed } \\
\text { (spire) }\end{array}$ & $\begin{array}{l}\text { DEM input } \\
\text { (spire) }\end{array}$ & $\begin{array}{l}\text { Predicted } \\
\text { (spire) }\end{array}$ & $\begin{array}{l}\text { Predicted from AIA DEM } \\
\text { (spire) }\end{array}$ & $\begin{array}{c}\text { Pre-jet } \\
\text { (footpoint) }\end{array}$ & $\begin{array}{l}\text { Observed } \\
\text { (footpoint) }\end{array}$ & $\begin{array}{l}\text { DEM input } \\
\text { (footpoint) }\end{array}$ & $\begin{array}{l}\text { Predicted } \\
\text { (footpoint) }\end{array}$ & $\begin{array}{l}\text { Predicted from AIA DEM } \\
\text { (footpoint) }\end{array}$ \\
\hline 94 & 0.5 & 1.1 & 0.6 & $0.2(2.7)$ & $0.6(1.0)$ & 1.8 & 6.4 & 4.6 & $3.9(1.1)$ & $4.6(1.0)$ \\
\hline 131 & 3.3 & 11.5 & 8.2 & $6.8(1.2)$ & $8.2(1.0)$ & 16.2 & 47.2 & 31 & $31.4(1.0)$ & $32.1(1.0)$ \\
\hline 171 & 194.7 & 294.2 & 99.5 & $120(0.8)$ & $98(1.0)$ & 457 & 769.4 & 312.7 & $305.1(1.0)$ & $303(1.0)$ \\
\hline 193 & 86 & 151 & 65 & $72(0.9)$ & $67(1.0)$ & 340.1 & 802.3 & 462.2 & 430 (1.1) & 442 (1.1) \\
\hline 211 & 22.2 & 52.4 & 30.2 & $28.5(1.1)$ & $29(1.02)$ & 123 & 291 & 168.1 & $184.2(0.9)$ & $180(0.9)$ \\
\hline 335 & 0.6 & 1.7 & 1.1 & $1.4(0.8)$ & $1.0(1.0)$ & 4.2 & 11.4 & 7.1 & $8.0(0.9)$ & $6.7(1.1)$ \\
\hline Si IV & & & 4927.9 & $2886(1.7)$ & & & & 10182 & $8652(1.2)$ & \\
\hline O IV & & & 1093.0 & $1063(1.02)$ & & & & 1258 & $1302(1.0)$ & \\
\hline Fe XVIII & & & & & & & & 2.1 & $2.4(0.9)$ & \\
\hline
\end{tabular}

Notes. Columns 3 and 8 indicate the observed intensities (DN/s) from the time-averaged AIA channels obtained from Fig. 8 for the spire and footpoint region respectively. Columns 2 and 7 indicate the observed intensities (DN/s) for the pre-jet phase. Column 4 and 9 indicate the AIA intensities obtained after subtraction of pre-jet intensities from the observed intensities. The intensities obtained from the Si IV and O IV lines are in erg cm${ }^{-2} \mathrm{~s}^{-1} \mathrm{sr}^{-1}$ units. Columns 5 and 10 indicate the predicted intensities obtained from the AIA-IRIS DEM analysis. Columns 6 and 11 indicate the predicted intensities obtained from the DEM analysis using only AIA data. The observed to predicted intensity ratio for each channel is given in parenthesis.
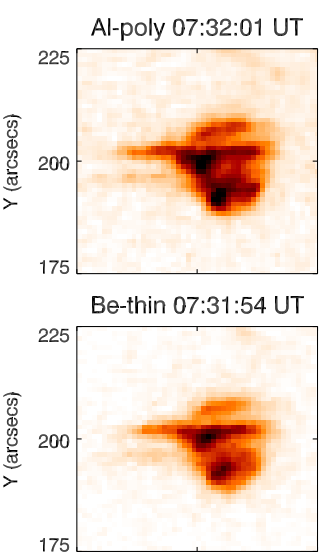

XRT temp map

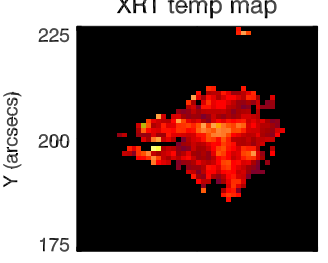

60
07:36:32 UT

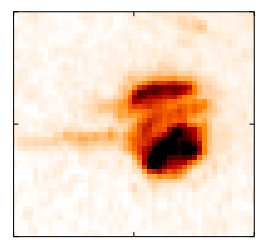

07:36:24 UT
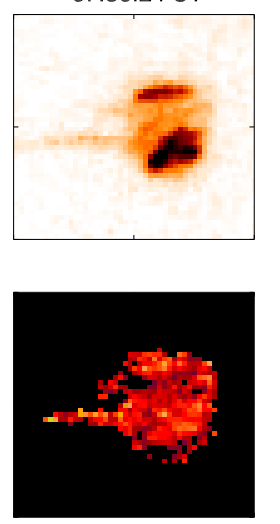

$85 \quad 11060$
07:39:32 UT

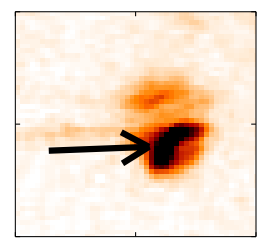

07:39:24 UT
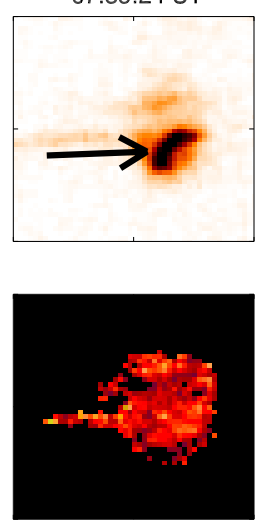

85
07:50:00 UT

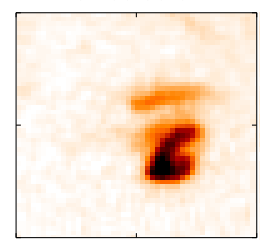

07:49:53 UT
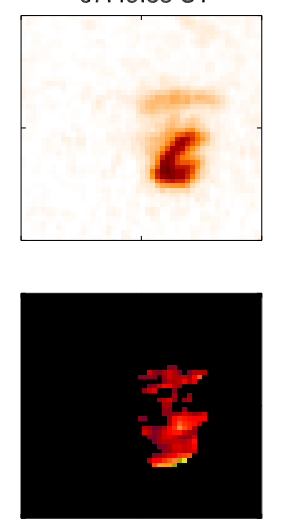

85

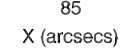

X (arcsecs)

11060

$X(\operatorname{arcsecs})$

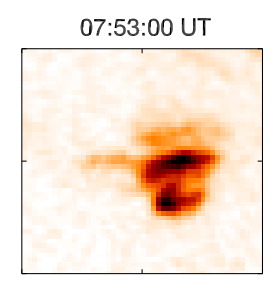

07:52:53 UT
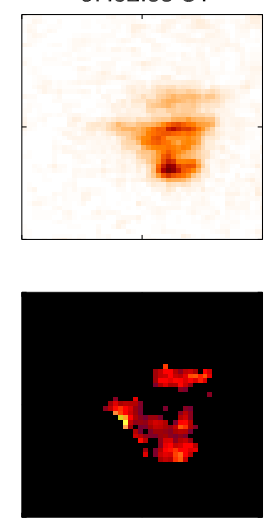

85

X (arcsecs)
08:34:00 UT

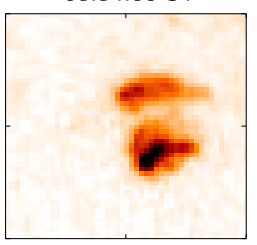

08:33:52 UT
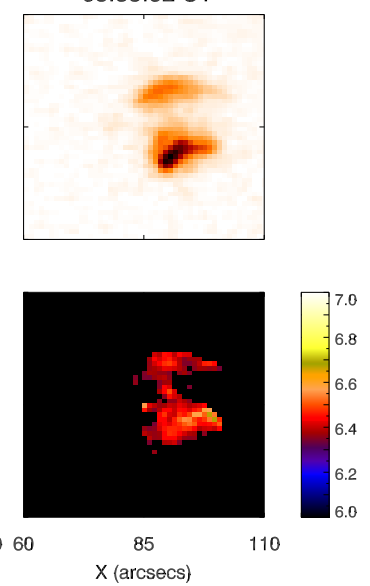

Fig. 11. XRT images and temperature maps during the recurring jet activity. The XRT Al-poly (top panel), Be-thin (middle panel) images (reverse colour) and XRT temperature maps (bottom panel) of the recurrent jet activity. The colourbar indicates XRT temperature in logarithmic scale. The black arrows (third column from the left) indicate the small loop-like structure inside the foopoint region of the jet. The image in first column (from left) shows jet 1,4 th and 5 th columns show activity of jet 2 and 6 th column shows later phase of jet 4 .

footpoint region appears to be multithermal at 07:39 UT (shown by black arrows). At later times, the Fe XVIII emission was only present at a few small locations in the footpoint region as shown in Fig. 12 (Cols. 3-6). Overall, in the jet footpoint, the hot and cool emission appears to be co-spatial and co-temporal, within the resolution/cadence of the observations.

Given the rapid evolution of the jets, a proper analysis of the different structures within the same jet would require combining information from the SJI images with the AIA ones. We investigated whether the SJI images could be used directly for quantitative analysis (e.g. DEM), rather than just morphological studies. This is a complex issue that has not been addressed in the literature, as far as we are aware. In principle, the SJI C II and $\mathrm{Si}$ IV bands are centered on the strong lines from these two ions, so if the emission from these lines were dominating the band, the SJI images could be used directly to estimate the cool emission in
C II and Si IV. Unfortunately it turns out that, given the current understanding of the IRIS calibration, this does not seem to be the case, as the bands are relatively broad. A detailed study of these channels will be presented in a future paper.

\section{Discussion and summary}

In this paper, we have studied a recurrent AR jet observed on July 10, 2015 using the IRIS, AIA and XRT instruments. We performed an in-depth analysis of the temperature structure of the spire and footpoint regions of the jet by combining the IRIS spectra with the AIA images in the DEM analysis. The purpose of this analysis is to get a better constraint on the lower temperatures for the DEMs by including the IRIS Si IV and O IV lines.

For the spire region, we found significant emission at cool temperatures, with a significant increase down to $\log T[\mathrm{~K}]=4.1$. 
The spire DEM shows a plateau in the temperature range from $\log T[\mathrm{~K}]=5.6$ to 5.9. Similarly, the footpoint region had strong cool emission, while the hot emission peaked at $\log T[\mathrm{~K}]=6.5$. The total EM for the footpoint region is one order of magnitude higher than in the spire. We calculated a filling factor of 0.1 in the spire region of the jet 4 for emission at $\sim 10^{5} \mathrm{~K}$. We also calculated the EM in both regions using only AIA data in the temperature range $5.2<\log T[\mathrm{~K}]<7.0$ and found that the values are almost three orders of magnitude lower than those obtained by combining AIA and IRIS in the DEM analysis (see Table 3). The predicted intensities from the DEM curves show good agreement with the observed ones in both regions (see Table 4).

The presence of plasma at $T>2 \mathrm{MK}$ in the footpoint region was confirmed by estimating the Fe XVIII emission. However, the bulk of the hot plasma had an effective temperature of only $T \sim 3 \mathrm{MK}$ in the footpoint region. The average temperature of the footpoint did not show any significant change over the period of time during jet 1 and 2 and also a similar temperature was obtained in the later phase of jet 4 activity when the XRT observations were available. We believe that the temperature did not change significantly during the main phase of jet 4 and shows consistency with the results obtained from the DEM analysis.

The electron densities were obtained from the O IV $(1399 / 1401 \AA)$ density diagnostics in the spire $\left(2.0 \times 10^{10} \mathrm{~cm}^{-3}\right)$ and footpoint $\left(7.6 \times 10^{10} \mathrm{~cm}^{-3}\right)$ regions. These results are close to those obtained by Cheung et al. (2015). We obtained the planeof-sky velocities for the jet 2 and jet 4 in the transition region using the SJI Si IV images and in the lower corona using the AIA $171 \AA$ channel. Jet 4 showed higher velocities compared to jet 2 velocities in the lower corona and also in the transition region (see Table 2). The Doppler velocity in the spire region of the jet $4\left(32 \mathrm{~km} \mathrm{~s}^{-1}\right)$ was also found to be higher than the other three jets. In the footpoint region, the Doppler velocities for all jets were very similar. The Doppler velocities obtained here were found to be smaller than the results obtained by Cheung et al. (2015). The nonthermal velocities were found to be higher in the spire regions of all jets than in the foopoint regions. The nonthermal velocity was higher in the spire region of jet 2 and jet 4 compared with the other two jets. This might be because of the untwisting nature of the spire structure and plasma motion along different threads of the spire.

The evolution of the footpoint of the recurrent jets was examined using the Fe XVIII and the SJI Si IV images. The results confirmed the presence of co-spatial and co-temporal multi-temperature plasma in the footpoint region of the jets (see Fig. 12).

Brueckner \& Bartoe (1983) reported an analysis of four high energy jets in the quiet Sun region observed by the High Resolution Telescope and Spectrograph (HRTS) experiment. The jets were observed over the range of temperatures from $2 \times 10^{4}$ to $2 \times 10^{5} \mathrm{~K}$. The authors studied the plasma parameters such as total EM $\left(6.0 \times 10^{27}-1.5 \times 10^{28} \mathrm{~cm}^{-5}\right)$, electron number density $\left(7 \times 10^{9} \mathrm{~cm}^{-3}\right)$, Doppler velocities $\left(50-400 \mathrm{~km} \mathrm{~s}^{-1}\right)$. We found close agreement between our results and those obtained with HRTS, although our jets occurred within an active region, and our densities are somewhat higher.

Liu et al. (2016) reported a very high temperature ( 16 MK) for one of the jets which originated from the same active region on July 09, 2015. The same authors estimated the DEM using the AIA and derived an electron number density of $0.7-1.1 \times 10^{9} \mathrm{~cm}^{-3}$ from the AIA emission measures. They used these values to provide various estimates of mass, and energy budget for the jets.
By combining the IRIS, AIA and XRT observations, we can rule out the presence of such high temperatures $(\sim 16 \mathrm{MK})$ for the jets we have analysed. We have also shown that by using only AIA, the total emission measure for the jets is typically underestimated by three orders of magnitudes (see Table 3 ). The densities found by Liu et al. (2016) are much lower by almost one order of magnitude, compared to those we obtained for the cool emission. Therefore, the estimates of the mass and energy for these jets should take into account the cool emission.

Mulay et al. (2016) perfomed a multiwavelength study of 20 AR jets using AIA observations. The authors obtained DEMs using only the AIA EUV channels and calculated peak temperature (spire and footpoint: $\log T[\mathrm{~K}]=6.2-6.3$ and 6.5 respectively), EM $\left(1-4 \times 10^{28} \mathrm{~cm}^{-5}\right)$ and the electron number density $\left(N_{\mathrm{e}}=8.4 \times 10^{9}-1.3 \times 10^{10} \mathrm{~cm}^{-3}\right)$ in the spire and footpoint region. We compared our results (those obtained using only AIA channels) with their results. We found that the peak temperature and the EM for the spire show smaller values whereas in case of footpoint, peak temperature and the EM values show good agreement. The plane-of-sky velocities obtained using the AIA $171 \AA$ channel show good agreement with the results obtained in the 20 jet's study.

Mulay et al. (2017) performed a DEM analysis of a recurrent AR jet separately using the EIS spectroscopic observation and also by combining the AIA and XRT observations. This procedure better constrained the high temperature DEM values by adding XRT data, but the low temperature DEM values were not constrained in the EIS DEM and AIA-XRT DEM. The authors found a peak temperature $T=2.0 \mathrm{MK}(\log T[\mathrm{~K}]=6.3)$ in the spire and the footpoint region. This is higher in the spire region compared to the results we obtained by combining AIA and IRIS and in the footpoint region, it shows good agreement. They calculated the $\operatorname{EM}\left(2.2-3.5 \times 10^{28} \mathrm{~cm}^{-5}\right)$ and electron number densities $\left(N_{\mathrm{e}}=7.6 \times 10^{10}-1.1 \times 10^{11} \mathrm{~cm}^{-3}\right.$ at $\left.\log T[\mathrm{~K}]=6.2\right)$ in the spire and the footpoint region. Using the AIA and IRIS together, we obtained EM values which are found to be two orders of magnitude higher in the spire region and three orders of magnitude higher in the footpoint compared to those obtained in Mulay et al. (2017). The electron pressure obtained using O IV in this paper is found to be one order of magnitude lower compared with their values obtained from the Fe XII.

Our results provide observational evidence for the coexistence of cool and hot plasma in the recurring AR jets. These results resemble the scenario proposed by Yokoyama \& Shibata (1996) and Nishizuka et al. (2008) in their 2D numerical simulation of coronal hole jets. Yokoyama \& Shibata (1996) reported a complex distribution of plasma ranging from $T \approx 10^{4}$ (cool jets) to $10^{6} \mathrm{~K}$ (hot jets). These temperatures are found to be lower than those compared with our observations. Nishizuka et al. (2008) used more realistic conditions for the corona and reported temperature of $10^{5}$ for the cool jets and very high temperature $\left(>5 \times 10^{6}-10^{7} \mathrm{~K}\right)$ in hot jets. Our results show good agreement with their results in case of cool jets, but they obtained much higher temperatures in the coronal components of the jets.

Moreno-Insertis et al. (2008) carried out a 3D numerical simulations of flux emergence in a coronal hole and found good agreement with observations from XRT and EIS instruments. They calculated high blue-shifts of $240 \mathrm{~km} \mathrm{~s}^{-1}$, an electron number density of $5.5 \times 10^{9} \mathrm{~cm}^{-3}$ at $\log T[\mathrm{~K}]=6.2$ and a filling factor of 0.2 . From the simulation, they reported velocity above 200 $\mathrm{km} \mathrm{s}^{-1}$, densities about 10 times the density of the ambient corona and temperatures of the jet above $10^{7} \mathrm{~K}$. They also observed ejection of cool and dense plasma from the reconnection site in the 

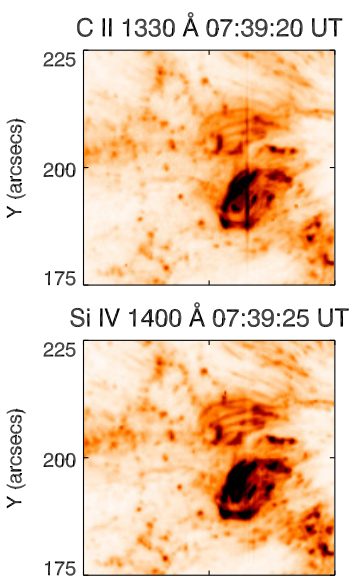

Fe XVIII 07:39:25 UT
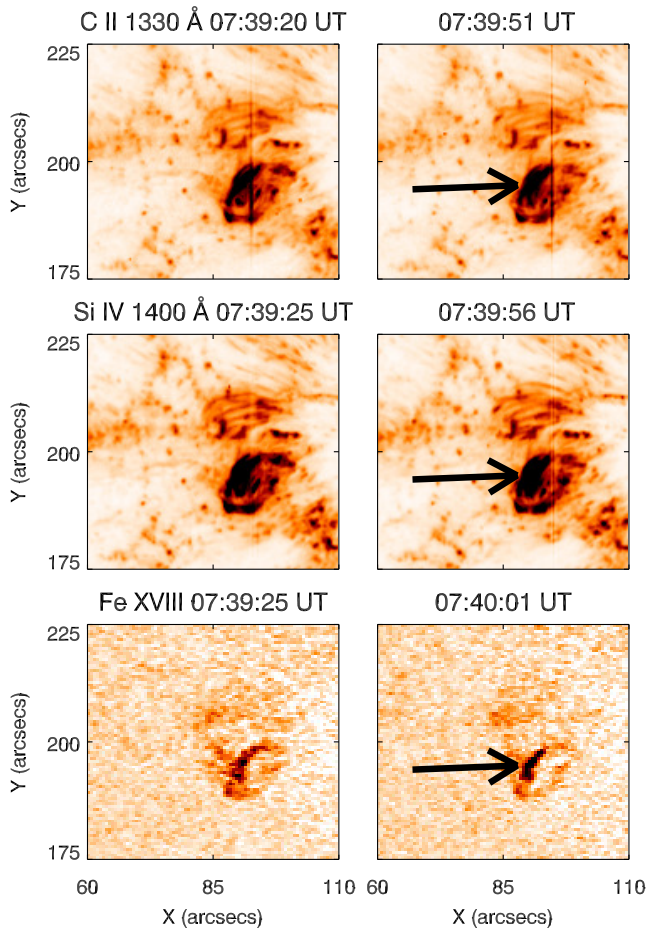

07:39:56 UT

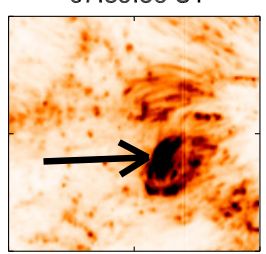

07:40:01 UT

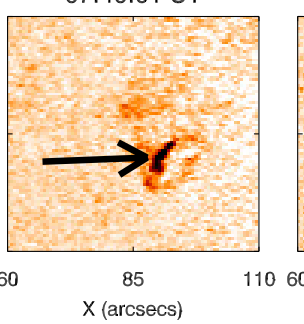

07:53:00 UT

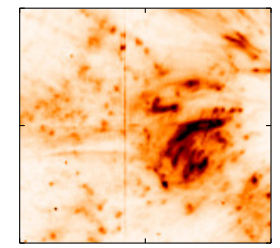

07:53:05 UT

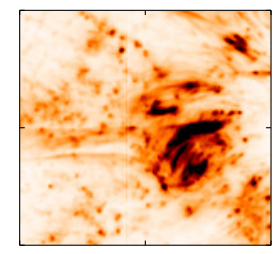

07:53:01 UT

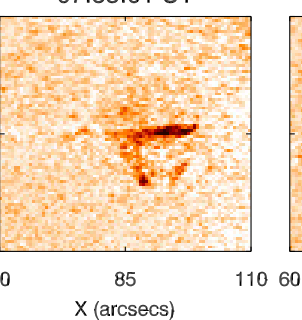

08:21:05 UT

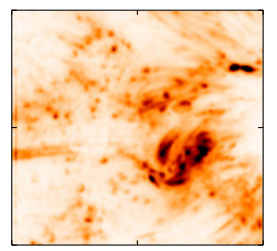

08:21:11 UT

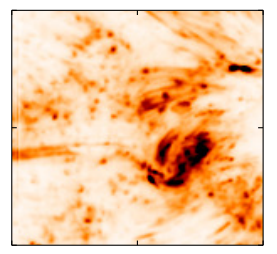

08:21:13 UT

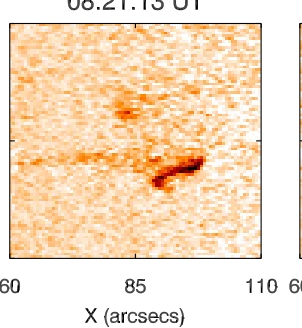

08:22:54 UT

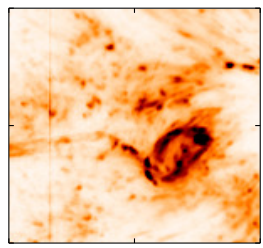

08:22:59 UT

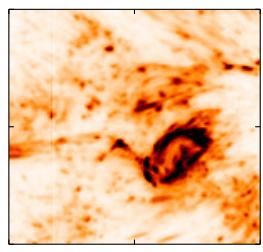

08:23:01 UT

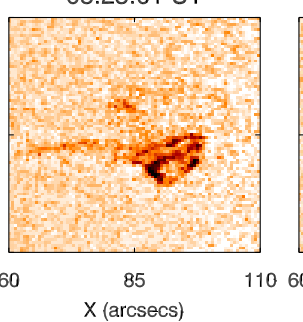

08:25:59 UT

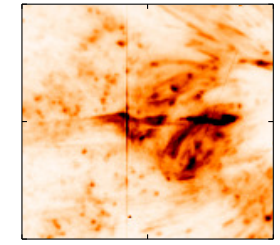

08:26:04 UT

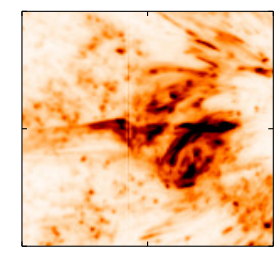

08:26:01 UT

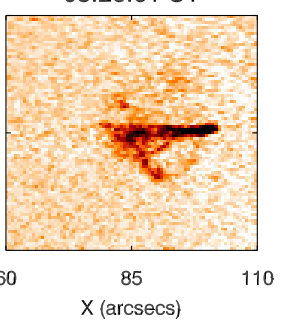

Fig. 12. Cool (C II and Si IV) and hot (Fe XVIII) components of jets. The SJI C II $1330 \AA$ A (top panel), Si IV $1400 \AA$ (middle panel) and the Fe XVIII images (bottom panel) (reverse colour) during the recurrent jet activity. The black arrows (second column from the left) indicate the small loop-like structure inside the foopoint region of the jet. The third column sampled jet 2, the fourth to sixth column sampled jet 4.

early stages of the reconnection. The electron pressure is found to be in good agreement with our results. The evolution of this jet was studied in detail with 3D simulation by Moreno-Insertis $\&$ Galsgaard (2013). The authors reported low densities about $10^{9} \mathrm{~cm}^{-3}$, velocities from 100 to $300 \mathrm{~km} \mathrm{~s}^{-1}$ and very high temperature about $10^{7} \mathrm{~K}$. The velocities are close to the values we obtained, but the temperature is very high.

To the best of our knowledge there are very few numerical studies (Gontikakis et al. 2010; Archontis et al. 2010) available for the AR jets. Gontikakis et al. (2009) and Gontikakis et al. (2010) obtained good qualitative and quantitative agreement between AR jet observation and 3D MHD numerical simulations of magnetic flux emergence in an active region. Using the Solar Ultraviolet Measurements of Emitted Radiation (SUMER) instrument, the authors reported an electron density of $2 \times 10^{9} \mathrm{~cm}^{-3}$ and a temperature of the jet ranging from $10^{5}$ to $1.5 \times 10^{6} \mathrm{~K}$. The authors calculated Doppler velocities of $-100 \mathrm{~km} \mathrm{~s}^{-1}$ (blueshift) and $40 \mathrm{~km} \mathrm{~s}^{-1}$ (redshift) at a temperature of $10^{5.8} \mathrm{~K}$ (Ne VIII $770 \AA$ ) and $-20 \mathrm{~km} \mathrm{~s}^{-1}$ (blueshift) and $33 \mathrm{~km} \mathrm{~s}^{-1}$ (redshift) at temperature of $10^{5} \mathrm{~K}$ (C IV $1548 \AA$ ). The Doppler velocities we obtained show smaller values compared with those obtained with Ne VIII $770 \AA$ line but they are close to the values obtained from the C IV $1548 \AA$ line. The electron pressure is found to be in good agreement with our results.

In the 3D numerical simulation (Gontikakis et al. 2010) of AR jets, the velocity was recorded to be around $100 \mathrm{~km} \mathrm{~s}^{-1}$. The temperature in the jet spire reached up to $1 \mathrm{MK}$ and in the footpoint region, it reached up to $2 \mathrm{MK}$. Their temperature is high in the spire but low in the footpoint compared with our observations.

The long term evolution of the same simulation in 3D was studied further by Archontis et al. (2010). They reported successive episodes of recurrent jets in direction perpendicular to each other. They noted velocities of 100-200 $\mathrm{km} \mathrm{s}^{-1}$, temperatures of a few MK and density of 10 times the ambient density for the first instance of a jet. These physical parameters were found to be decreasing with successive ejection of jets and eventually the system reached an equilibrium. Our coronal jet temperature agrees with the results they obtained but all recurrent jets were observed to originate in the same direction. We obtained higher velocities in jets than reported in their simulation. Neither AR simulations show the presence of cool jet $\left(10^{5} \mathrm{~K}\right)$ during the activity.

The possible interpretation for the co-existence of cool and hot plasma in the recurring AR jets could be the magnetic reconnection which occurred in the transition region or upper chromosphere. We believe that the results shown in this paper might be helpful for the theoretical modeling and also to produce physical plasma parameters for the AR jets in 2D and 3D numerical simulations.

Acknowledgements. We thank the referee for constructive and detailed comments which helped to improve this paper. S.M.M. acknowledges support from the Cambridge Trust, University of Cambridge, UK. H.E.M. and G.D.Z. acknowledge the support of STFC. The authors also thank Dr. P. Kayshap, Dr. D. Tripathi (IUCAA) and Dr. H. Isobe for useful discussions. We also thank the IRIS team (in particular Jean-Pierre Wuelser and Wei Liu) for sharing information about the FUV background and the IRIS calibration. AIA data are courtesy of SDO (NASA) and the AIA consortium. CHIANTI is a collaborative project involving George Mason University, the University of Michigan (USA) and the University of Cambridge (UK). Hinode is a Japanese mission developed and launched by ISAS/JAXA, with NAOJ as domestic partner and NASA and STFC (UK) as international partners. It is operated by these agencies in co-operation with ESA and NSC (Norway). IRIS is a NASA small explorer mission developed and operated by LMSAL with mission operations executed at NASA Ames Research center and major contributions to downlink communications funded by ESA and the Norwegian Space Centre.

\section{References}

Adams, M., Sterling, A. C., Moore, R. L., \& Gary, G. A. 2014, ApJ, 783, 11 Archontis, V., Tsinganos, K., \& Gontikakis, C. 2010, A\&A, 512, L2

Aschwanden, M. J., Boerner, P., Schrijver, C. J., \& Malanushenko, A. 2013, Sol. Phys., 283, 5 
Asplund, M., Grevesse, N., Sauval, A. J., \& Scott, P. 2009, ARA\&A, 47, 481 Brueckner, G. E., \& Bartoe, J.-D. F. 1983, ApJ, 272, 329

Canfield, R. C., Reardon, K. P., Leka, K. D., et al. 1996, ApJ, 464, 1016

Chandra, R., Gupta, G. R., Mulay, S., \& Tripathi, D. 2015, MNRAS, 446, 3741

Chandrashekhar, K., Morton, R. J., Banerjee, D., \& Gupta, G. R. 2014, A\&A, 562, A98

Chen, H. D., Jiang, Y. C., \& Ma, S. L. 2008, A\&A, 478, 907

Chen, N., Ip, W.-H., \& Innes, D. 2013, ApJ, 769, 96

Cheung, M. C. M., De Pontieu, B., Tarbell, T. D., et al. 2015, ApJ, 801, 83

Chifor, C., Isobe, H., Mason, H. E., et al. 2008a, A\&A, 491, 279

Chifor, C., Young, P. R., Isobe, H., et al. 2008b, A\&A, 481, L57

Cirtain, J. W., Golub, L., Lundquist, L., et al. 2007, Science, 318, 1580

Culhane, J. L., Harra, L. K., James, A. M., et al. 2007a, Sol. Phys., 243, 19

Culhane, L., Harra, L. K., Baker, D., et al. 2007b, PASJ, 59, S751

De Pontieu, B., Title, A. M., Lemen, J. R., et al. 2014, Sol. Phys., 289, 2733

Del Zanna, G. 2013, A\&A, 558, A73

Del Zanna, G., O'Dwyer, B., \& Mason, H. E. 2011, A\&A, 535, A46

Del Zanna, G., Dere, K. P., Young, P. R., Landi, E., \& Mason, H. E. 2015, A\&A, 582, A56

Dere, K. P., Landi, E., Mason, H. E., Monsignori Fossi, B. C., \& Young, P. R. 1997, A\&AS, 125, 149

Freeland, S. L., \& Handy, B. N. 1998, Sol. Phys., 182, 497

Golub, L., Deluca, E., Austin, G., et al. 2007, Sol. Phys., 243, 63

Gontikakis, C., Archontis, V., \& Tsinganos, K. 2009, A\&A, 506, L45

Gontikakis, C., Archontis, V., \& Tsinganos, K. 2010, in 9th Int. Conf. Hellenic Astronomical Society, eds. K. Tsinganos, D. Hatzidimitriou, \& T. Matsakos, ASP Conf. Ser., 424, 19

Hannah, I. G., \& Kontar, E. P. 2012, A\&A, 539, A146

Hong, J., Jiang, Y., Yang, J., Li, H., \& Xu, Z. 2017, ApJ, 835, 35

Hong, J., Jiang, Y., Zheng, R., et al. 2011, ApJ, 738, L20

Hong, J.-C., Jiang, Y.-C., Yang, J.-Y., et al. 2013, Res. Astron. Astrophys., 13, 253

Innes, D. E., Cameron, R. H., \& Solanki, S. K. 2011, A\&A, 531, L13

Innes, D., Bucik, R., Guo, L.-J., \& Nitta, N. 2016, Astron. Narch., 337, 1024

Jiang, Y. C., Chen, H. D., Li, K. J., Shen, Y. D., \& Yang, L. H. 2007, A\&A, 469, 331

Joshi, N. C., Chandra, R., Guo, Y., et al. 2017, Ap\&SS, 362, 10

Kamio, S., Hara, H., Watanabe, T., et al. 2007, PASJ, 59, S757

Kayshap, P., Srivastava, A. K., \& Murawski, K. 2013, ApJ, 763, 24

Kim, Y.-H., Moon, Y.-J., Park, Y.-D., et al. 2007, PASJ, 59, S763

Kobelski, A. R., Saar, S. H., Weber, M. A., McKenzie, D. E., \& Reeves, K. K. 2014, Sol. Phys., 289, 2781

Kosugi, T., Matsuzaki, K., Sakao, T., et al. 2007, Sol. Phys., 243, 3

Lemen, J. R., Title, A. M., Akin, D. J., et al. 2012, Sol. Phys., 275, 17

Liu, J., Wang, Y., Erdélyi, R., et al. 2016, ApJ, 833, 150
Martínez Pillet, V., Del Toro Iniesta, J. C., \& Quintero Noda, C. 2011, A\&A, 530, A111

Moreno-Insertis, F., \& Galsgaard, K. 2013, ApJ, 771, 20

Moreno-Insertis, F., Galsgaard, K., \& Ugarte-Urra, I. 2008, ApJ, 673, L211

Mulay, S. M., Tripathi, D., Del Zanna, G., \& Mason, H. 2016, A\&A, 589, A79

Mulay, S. M., Zanna, G. D., \& Mason, H. 2017, A\&A, 598, A11

Narukage, N., Sakao, T., Kano, R., et al. 2011, Sol. Phys., 269, 169

Nishizuka, N., Shimizu, M., Nakamura, T., et al. 2008, ApJ, 683, L83

Nisticò, G., Patsourakos, S., Bothmer, V., \& Zimbardo, G. 2011, Adv. Space Res., 48, 1490

O’Dwyer, B., Del Zanna, G., Mason, H. E., Weber, M. A., \& Tripathi, D. 2010, A\&A, 521, A21

Panesar, N. K., Sterling, A. C., \& Moore, R. L. 2016a, ApJ, 822, L23

Panesar, N. K., Sterling, A. C., Moore, R. L., \& Chakrapani, P. 2016b, ApJ, 832, L7

Polito, V., Reeves, K. K., Del Zanna, G., Golub, L., \& Mason, H. E. 2015, ApJ, 803,84

Polito, V., Del Zanna, G., Dudík, J., et al. 2016, A\&A, 594, A64

Raouafi, N. E., Patsourakos, S., Pariat, E., et al. 2016, Space Sci. Rev., 201, 1

Rubio da Costa, F., Solanki, S. K., Danilovic, S., Hizberger, J., \& Martínez-Pillet, V. 2015, A\&A, 574, A95

Schmieder, B., Guo, Y., Moreno-Insertis, F., et al. 2013, A\&A, 559, A1

Shen, Y., Liu, Y., Su, J., \& Ibrahim, A. 2011, ApJ, 735, L43

Shibata, K., Ishido, Y., Acton, L. W., et al. 1992, PASJ, 44, L173

Shibata, K., Nitta, N., Strong, K. T., et al. 1994, ApJ, 431, L51

Shibata, K., Nakamura, T., Matsumoto, T., et al. 2007, Science, 318, 1591

Shimojo, M., Hashimoto, S., Shibata, K., et al. 1996, PASJ, 48, 123

Sterling, A. C., Moore, R. L., Falconer, D. A., et al. 2016, ApJ, 821, 100

Sterling, A. C., Moore, R. L., Falconer, D. A., Panesar, N. K., \& Martinez, F. 2017, ApJ, 844, 28

Tsuneta, S., Acton, L., Bruner, M., et al. 1991, Sol. Phys., 136, 37

Wang, H., Johannesson, A., Stage, M., Lee, C., \& Zirin, H. 1998, Sol. Phys., 178, 55

Wang, J.-F., Zhou, T.-H., \& Ji, H.-S. 2014, Chin. Astron. Astrophys., 38, 65

Weber, M. A., Deluca, E. E., Golub, L., \& Sette, A. L. 2004, in Multi-Wavelength Investigations of Solar Activity, eds. A. V. Stepanov, E. E. Benevolenskaya, \& A. G. Kosovichev, IAU Symp., 223, 321

Yokoyama, T., \& Shibata, K. 1995, Nature, 375, 42

Yokoyama, T., \& Shibata, K. 1996, PASJ, 48, 353

Yoshimura, K., \& McKenzie, D. E. 2015, Sol. Phys., 290, 2355

Young, P. R., \& Muglach, K. 2014a, PASJ, 66, S12

Young, P. R., \& Muglach, K. 2014b, Sol. Phys., 289, 3313

Yu-kun, H., Zhi, X., Zhi-ke, X., et al. 2016, Chin. Astron. Astrophys., 40, 504

Zhang, Q. M., \& Ji, H. S. 2014a, A\&A, 561, A134

Zhang, Q. M., \& Ji, H. S. 2014b, A\&A, 567, A11 


\section{Appendix A: Spectra in the spire region}

The averaged calibrated spectra for spire region of the jet 4 (shown as small green box in Fig. 8) obtained by using the iris_sum_spec.pro routine.

\section{A.1. Pre-jet AIA images}

In order to measure the foreground emission in all AIA channels, we chose near-simultaneous images at 08:10:35 UT before the jet 4 activity. Figure A. 2 shows AIA images in six coronal channels. The spire and footpoint regions are shown with green and yellow boxes respectively. The averaged intensities in these regions were subtracted from the observed intensities (obtained from Fig. 8) in the spire and footpoint region of jet 4 and then used as an input for the DEM analysis.

\section{A.2. Modification in the DEM program}

We modified the xrt_dem_iterative2.pro DEM inversion routine (Weber et al. 2004) to calculate DEMs by combining the IRIS and AIA observations. We simplified the program, which ultimately uses the chi-square fitting routine (mpfit . pro) to reduce the chi-square between the actual and model DEM. The DEM is modelled assuming a spline. We modified the program by allowing a definition of the spline nodes, and allowing to input minimum and maximum limits to the spline values, which are passed to mpfit.pro. We took parameters of equally spaced seven spline knots. We provided the observed AIA DN/s and relative responses as an input, combined with the calibrated C II and $\mathrm{Si}$ IV radiances and relative contribution functions. Finally, we obtained the best DEM solution and a range of solutions by randomly varying the observed intensities within a $20 \%$ uncertainty.

\section{A.3. XRT temperature responses}

The XRT temperature responses that were used in the temperature calculations (refer Sect. 3.9) are shown in Fig. A.3.

\section{A.4. DEM of the foopoint region}

We perfomed a DEM analysis (as in Sect. 3.6) on another nearby region in the footpoint. Figure A.4 shows the time-averaged (reverse colours) image of the AIA $94 \AA$ channel (left panel) and rebinned calibrated Si IV raster image (right panel). The new region in the foopoint is shown with white boxes. We recorded the start time for the slit corresponding to the boxed region was 08:29:07 UT and the end time was 08:29:43 UT.

Figure A.5 show the DEM curve for the new footpoint region shown in Fig. A.4. The overplotted colour bars show 50\% (blue), $80 \%$ (red) and $95 \%$ (yellow) of the $300 \mathrm{MC}$ solutions closest to the best-fit DEM. The DEM curve show similar shape (see Fig. 9) as the DEM obtained for the foopoint region shown in Fig. 8. The hot emission peak at $\log T[\mathrm{~K}]=6.5$ and total EM $9.7 \times 10^{31} \mathrm{~cm}^{-5}$. The peak temperature and EM values are also close to those obtained for the footpoint region discussed in Sect. 3.6.

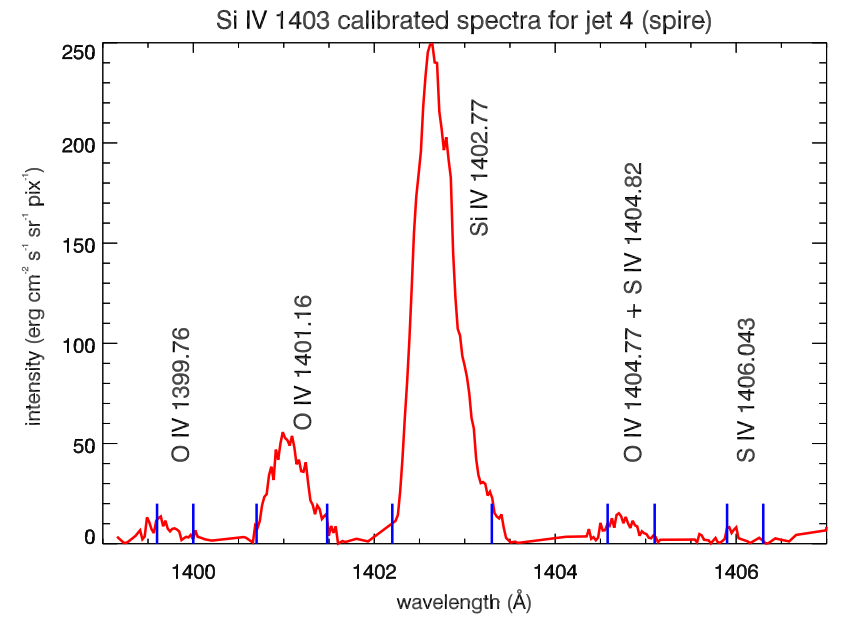

Fig. A.1. IRIS spectra for the spire region of the jet. The averaged calibrated spectra (in erg cm $\mathrm{cm}^{-1} \mathrm{sr}^{-1} \mathrm{pix}^{-1}$ units) obtained for the $\mathrm{Si}$ IV $1403 \AA$ window for the spire region of the jet observed in the fourth raster scan (shown as small green box in Fig. 8). The blue vertical lines show the wavelength ranges over which we obtained the total counts for each line.

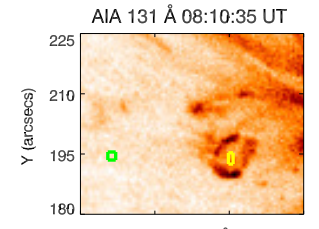

AIA $211 \AA$

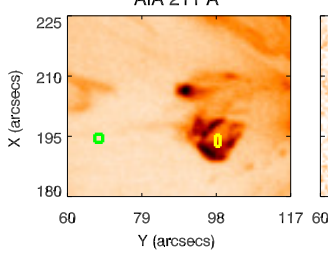

AIA $171 \AA$

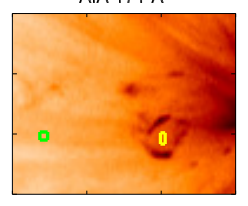

AIA $94 \AA$

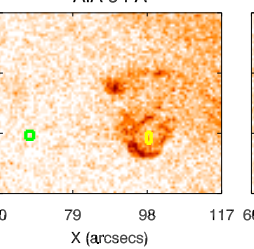

AIA $193 \AA$

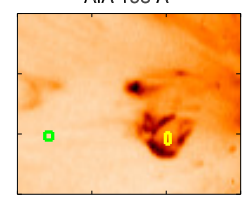

AIA $335 \AA$

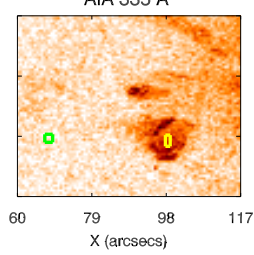

Fig. A.2. AIA images during the pre-jet phase. AIA (reverse colours) images in six coronal channels at 08:10:35 UT represent pre-jet phase of jet 4 . The green and yellow boxes indicate the region of the spire and the footpoint respectively (same as shown in Fig. 8).

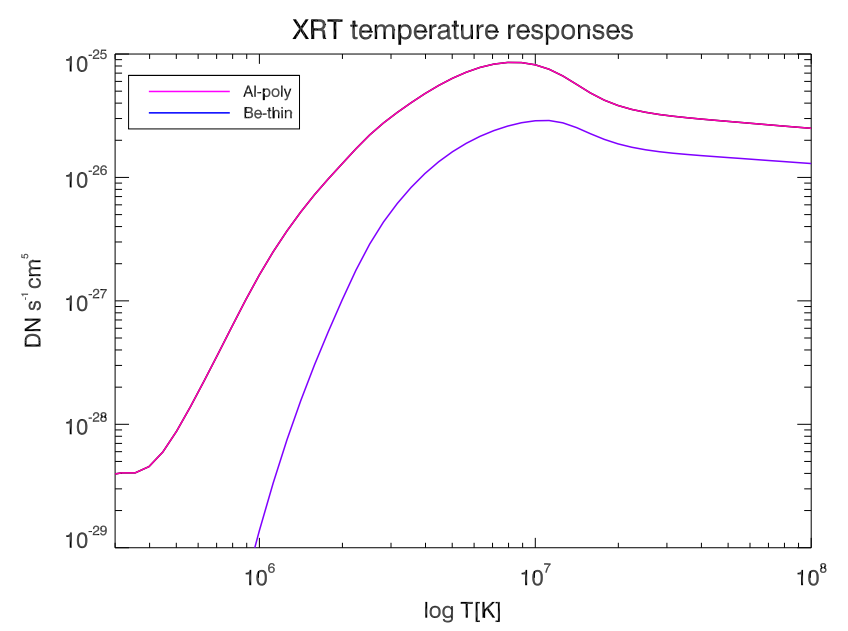

Fig. A.3. XRT temperature responses. The temperature resonses for the Al-poly and Be-thin filer of XRT instrument onboard Hinode satellite. We used the CHIANTI v.8 (Del Zanna et al. 2015) atomic database and the electron density of $N_{\mathrm{e}}=1 \times 10^{10} \mathrm{~cm}^{-3}$ and photospheric abundances by Asplund et al. (2009). 

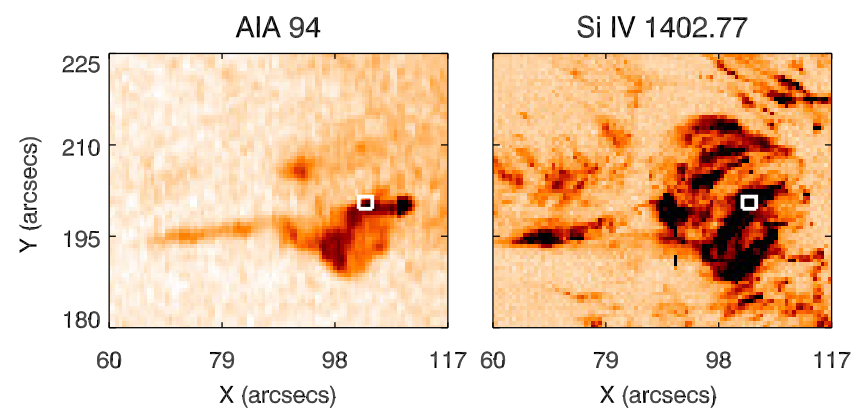

Fig. A.4. DEM input for another footpoint region. The time-averaged (reverse colours) image of the AIA $94 \AA$ channel (left panel) and rebinned calibrated Si IV raster image (right panel). The white overplotted boxes represent a region in the jet-footpoint where we perfomed the DEM analysis.

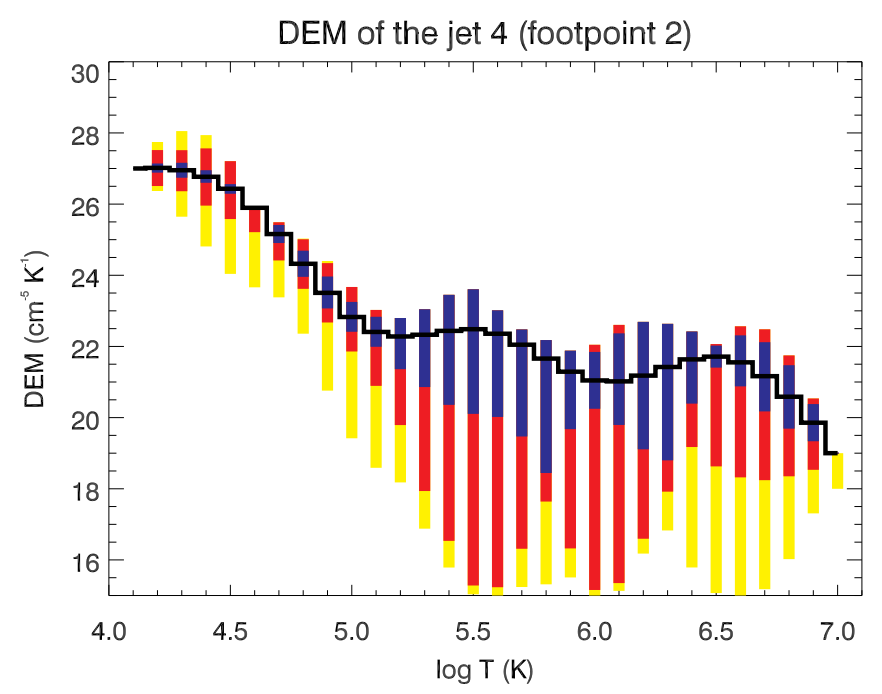

Fig. A.5. DEM result for another footpoint region. The DEM curve for the new footpoint region of the jet 4 shown in Fig. A.4. The overplotted colour bars show 50\% (blue), 80\% (red) and 95\% (yellow) of the 300 MC solutions.
Table A.1. Observed and predicted AIA and IRIS spectrograph intensities obtained from the DEM analysis for the new footpoint region of the jet 4 .

\begin{tabular}{|c|c|c|c|c|}
\hline (Col. 1) & (Col. 2) & (Col. 3) & (Col. 4) & (Col. 5) \\
\hline $\begin{array}{l}\text { Band } \\
(\AA)\end{array}$ & $\begin{array}{c}\text { Pre-jet } \\
\text { (footpoint) }\end{array}$ & $\begin{array}{c}\text { Observed } \\
\text { (footpoint) }\end{array}$ & $\begin{array}{l}\text { DEM input } \\
\text { (footpoint) }\end{array}$ & $\begin{array}{c}\text { Predicted } \\
\text { (footpoint) }\end{array}$ \\
\hline 94 & 1.1 & 6.8 & 5.7 & $3.4(1.6)$ \\
\hline 131 & 9.4 & 69.4 & 60 & $56.2(1.1)$ \\
\hline 171 & 480 & 964.3 & 484.2 & $482.6(1.0)$ \\
\hline 193 & 210 & 815.3 & 605.4 & $556.8(1.1)$ \\
\hline 211 & 54 & 223.2 & 170 & $188(1.0)$ \\
\hline 335 & 1.2 & 7.1 & 6.0 & $7.5(0.8)$ \\
\hline Si IV & & & 10921.3 & $6947(1.6)$ \\
\hline O IV & & & 1218.1 & $1294(1.0)$ \\
\hline
\end{tabular}

Notes. Column 3 indicates the observed intensities (DN/s) from the timeaveraged AIA channels obtained from Fig. A.4 for the new footpoint region. Column 2 indicates the observed intensities (DN/s) for the pre-jet phase. Column 4 indicates the AIA intensities obtained after subtraction of pre-jet intensities from the observed intensities. The intensities obtained from the Si IV and O IV lines are in erg $\mathrm{cm}^{-2} \mathrm{~s}^{-1} \mathrm{sr}^{-1}$ units. Column 5 indicates the predicted intensities obtained from the AIA-IRIS DEM analysis. The observed to predicted intensity ratio for each channel is given in parenthesis. 